\title{
HIDDEN MARKOV MODEL FOR PARAMETER ESTIMATION OF A RANDOM WALK IN A MARKOV ENVIRONMENT
}

\author{
Pierre Andreoletti ${ }^{1}$, Dasha Loukianova $^{2}$ and Catherine Matias $^{3}$
}

\begin{abstract}
We focus on the parametric estimation of the distribution of a Markov environment from the observation of a single trajectory of a one-dimensional nearest-neighbor path evolving in this random environment. In the ballistic case, as the length of the path increases, we prove consistency, asymptotic normality and efficiency of the maximum likelihood estimator. Our contribution is two-fold: we cast the problem into the one of parameter estimation in a hidden Markov model (HMM) and establish that the bivariate Markov chain underlying this HMM is positive Harris recurrent. We provide different examples of setups in which our results apply, in particular that of DNA unzipping model, and we give a simple synthetic experiment to illustrate those results.
\end{abstract}

Mathematics Subject Classification. 62M05, 62F12, 60J25.

Received November 19, 2014. Revised April 1, 2015.

\section{INTRODUCTION}

Random walks in random environments (RWRE) form a subclass of canonical models in the more general framework of random motions in random media that is widely used in physics. These models go back to the pioneer works of Chernov [16], who introduced them to describe DNA replication and of Temkin [39] who used them in the field of metallurgy. A more complete list of application fields may be found in the introduction [12] as well as in the references therein. These models have been intensively studied in the last four decades, mostly in the physics and probability theory literature. Some surveys on the topic include $[27,35,36,40]$.

Statistical issues raised by those processes have been overlooked in the literature until very recently, when new biophysics experiments produced data that can be modeled (at least in an ideal-case setup) by RWRE [7]. Consequently, a new series of works appeared on statistical procedures aiming at estimating parameters from RWRE data. Another motivation to these studies comes from the fact that RWRE are closely linked to branching processes with immigration in random environments (BPIRE) and that the statistical issues raised in one context may potentially have an impact in the other.

Keywords and phrases. Hidden Markov model, Markov environment, maximum likelihood estimation, random walk in random environment.

1 Laboratoire MAPMO, UMR CNRS 6628, Fédération Denis-Poisson, Université d'Orléans, Orléans, France.

Pierre.Andreoletti@univ-orleans.fr

2 Laboratoire de Mathématiques et Modélisation d'Évry, Université d'Évry Val d'Essonne, UMR CNRS 8071, Évry, France. dasha.loukianova@maths. univ-evry.fr

3 Laboratoire de Probabilités et Modèles Aléatoires, UMR CNRS 7599, Université Pierre et Marie Curie, Université Paris

Diderot, Paris, France. catherine.matias@math.cnrs.fr 
In [5], the author investigates the local time of the one dimensional recurrent RWRE in order to estimate the trajectories of the underlying random potential. In [6], the ideal-case model from [7] is considered: a (finite length) DNA molecule is unzipped several times and some device translates these unzippings into random walks along the DNA molecule, whose sequence of bases is the random environment. There, the goal is to reconstruct this environment and thus achieve sequencing of the molecule. They prove that a Bayesian estimator (maximum a posteriori) of this sequence of bases is consistent as the number of unzippings increases and they characterize the probability of reconstruction error. In a different setting, several authors have considered the information on the environment that is contained in one single trajectory of the walk with infinite length. In their pioneer work [1], Adelman and Enriquez consider a very general RWRE and provide equations linking the distribution of some statistics of the trajectory to some moments of the environment distribution. In the specific case of a onedimensional nearest neighbor path, those equations give moment estimators for the environment distribution parameters. More recently, the article [18] studies a maximum likelihood estimator (MLE) in the specific context of a one-dimensional nearest neighbor path in transient ballistic regime. The authors prove the consistency of this estimator (as the length of the trajectory increases). From a numerical point of view, this MLE outperforms the moment estimator constructed from [1]. In a companion article [25], they have further studied the asymptotic normality of the MLE (still in the ballistic regime), showed its asymptotic efficiency and constructed confidence intervals for the parameters. This work has been extended to the transient sub-ballistic regime in [24]. In this body of work on maximum likelihood procedures, the results rely on the branching structure of the sequence of the number of left steps performed by the walk, which was originally observed in [29]. In the recurrent case, as the walk visits every sites infinitely often, this branching process of left steps explodes and the same approach is useless there. In theory, it is possible in this case to estimate the environment itself at each site, and then show that the empirical measure converges to its distribution. The problem with such a "naive" approach is the localization phenomena of recurrent RWRE, discovered by [37]: most of the sites visited by the RWRE will be extremely few visited, because the walk spends a majority of its time in the valleys of the potential [3,4]. This non uniformity is automatically handled with the approach followed in [17] and the authors establish consistency of two estimators, a MLE and a maximum pseudo-likelihood estimator.

We now stress the fact that all the previously mentioned statistical works but [6] are valid in the case of an environment composed of independent and identically distributed (i.i.d.) random variables. While very convenient, this assumption might be restrictive in some contexts, e.g. DNA modeling. In the present work, we investigate the statistical estimation of a parametric Markov environment from a single trajectory of a onedimensional nearest-neighbor path, when its length increases to infinity. We consider the case of a transient walk in the ballistic regime. Our contribution is twofold: first, we show how the problem is cast into the one of estimating the parameter of a hidden Markov model (HMM), or more specifically of a first-order autoregressive process with Markov regime. Indeed, the RWRE itself is not a HMM but the branching process of the sequence of left steps performed by the walk, is. Second, we prove that the bivariate Markov chain that defines the underlying autoregressive process is Harris positive and we exhibit its stationary distribution. As a consequence, we can rely on previously established results for these autoregressive processes with Markov regime [22] and thus obtain the consistency and asymptotic normality of the MLE for the original nearest-neighbor path in Markov environment.

Roughly speaking, an autoregressive model with Markov regime is a bivariate process where the first component forms a latent (unobserved) Markov chain while conditionally on this first component, the second one has the structure of an autoregressive process. These processes form a generalization of hidden Markov models (HMM), in which the first component remains a latent Markov chain, while the second forms a sequence of independent observations, conditionally on the first. HMM have been introduced in [8] with finite - latent and observed - state spaces. Statistical properties of the MLE in HMM form a rich literature; a non exhaustive list would start with the seminal work [8], include the developments $[10,11,20,22,26,28,30,31]$ and finish with the latest results in [21]. A general introduction to HMM may be found in the survey [23] and the book [14].

While it is often believed that autoregressive processes with Markov regime are straightforward generalizations of HMM (and this is indeed the case concerning e.g. algorithmic procedures), the statistical properties of these models are slightly more difficult to obtain, (see e.g. for model selection issues[15]). As for the convergence 
properties of the MLE, only the article [22] considers the autoregressive case (instead of HMM) explaining why we focus on their results in our context. It is also worth noticing that many of the previous results (with exception of [21]) require uniform positivity of the transition density of the latent Markov chain, which might not be satisfied in some applications (particularly in the case of an unbounded latent state space). As in our case, the latent state space corresponds to the environment state space and is included in $(0,1)$, we do not face such problems. Moreover, we stress that the results in [22] rely on rather weak assumptions (compared to previous results in $[11,28]$, on which they are partly built). As a consequence, the assumptions that we obtain on RWRE are also rather weak and will be satisfied in many contexts.

This article is organized as follows. Our one-dimensional nearest-neighbor path in Markov environment is described in Section 2.1. Then we explain why the direct likelihood approach may not be followed (Sect. 2.2) and cast the estimation problem as the one of parameter estimation in a hidden Markov model (Sect. 2.3). After having set the scene, we state the assumptions (on the RWRE) and results in Section 3. We prove that (under classical assumptions) the MLE is consistent and asymptotically normal. Section 4 illustrates our results: we start by explaining how the likelihood may be computed (Sect. 4.1), then we explore different examples and describe our assumptions in these cases (Sect. 4.2) and close the section with synthetic experiments on a simple example (Sect. 4.3). The proofs of our results are presented in Section 5. The main point is to establish that the bivariate Markov chain that underlies the HMM is positive Harris recurrent (Sect. 5.1). Then consistency, asymptotic normality and efficiency (i.e. the asymptotic variance is the inverse of the Fisher information) follow from [22] by proving that our assumptions on the RWRE imply theirs on the HMM (Sects. 5.2 and 5.3, respectively).

\section{Model DESCRIPTION}

\subsection{Ballistic random walk in a Markov environment}

We start this section by describing the random environment. Let $S$ be a closed subset of $(0,1)$ either finite, discrete or continuous, and $\mathcal{B}(S)$ the associated Borel $\sigma$-field. The environment is given by $\boldsymbol{\omega}=\left(\omega_{x}\right)_{x \in \mathbb{Z}} \in S^{\mathbb{Z}}$, a positive Harris recurrent, aperiodic and stationary Markov chain with values in $S$ and transition kernel $Q: S \times \mathcal{B}(S) \rightarrow[0,1]$. We suppose that the transition kernel $Q=Q_{\theta}$ depends on some unknown parameter $\theta$ and that $\theta$ belongs to some compact space $\Theta \subset \mathbb{R}^{q}$. Moreover, $Q_{\theta}$ is absolutely continuous either with respect to (w.r.t.) the Lebesgue measure on $(0,1)$ when $S$ is continuous or w.r.t. the counting measure when $S$ is discrete, with density denoted by $q_{\theta}$. We denote by $\mu_{\theta}$ the density of its associated stationary distribution. Let us denote by $\mathbf{P}^{\theta}$ the law of the environment $\boldsymbol{\omega}$ on $\left(S^{\mathbb{Z}}, \mathcal{B}\left(S^{\mathbb{Z}}\right)\right)$ and $\mathbf{E}^{\theta}$ the corresponding expectation.

Now, conditionally on the environment, the law of the random walk $\mathbf{X}=\left(X_{t}\right)_{t \in \mathbb{N}}$ is the one of the time homogeneous Markov chain on $\mathbb{Z}$ starting at $X_{0}=0$ and with transition probabilities

$$
\forall(x, y) \in \mathbb{Z}^{2}, \quad P_{\boldsymbol{\omega}}\left(X_{t+1}=y \mid X_{t}=x\right)=\left\{\begin{array}{cc}
\omega_{x} & \text { if } y=x+1, \\
1-\omega_{x} & \text { if } y=x-1, \\
0 & \text { otherwise }
\end{array}\right.
$$

The measure $P_{\boldsymbol{\omega}}$ on $\left(\mathbb{Z}^{\mathbb{N}}, \mathcal{B}\left(\mathbb{Z}^{\mathbb{N}}\right)\right)$ is usually referred to as the quenched law of walk $\mathbf{X}$. Note that this conditional law does not depend on the parameter $\theta$ but only on the environment $\boldsymbol{\omega}$ at the current site $x$. We also denote by $p_{a}(x, y)$ the corresponding transition density (w.r.t. to counting measure), namely

$$
\forall(x, y) \in \mathbb{Z}^{2}, \forall a \in S, \quad p_{a}(x, y)=a \mathbb{1}\{y=x+1\}+(1-a) \mathbb{1}\{y=x-1\},
$$

where $\mathbb{1}\{\cdot\}$ denotes the indicator function. Next we define the measure $\mathbb{P}^{\theta}$ on $S^{\mathbb{Z}} \times \mathbb{Z}^{\mathbb{N}}$ through

$$
\forall F \in \mathcal{B}\left(S^{\mathbb{Z}}\right), \forall G \in \mathcal{B}\left(\mathbb{Z}^{\mathbb{N}}\right), \quad \mathbb{P}^{\theta}(F \times G)=\int_{F} P_{\boldsymbol{\omega}}(G) d \mathbf{P}^{\theta}(\omega) .
$$

The second marginal of $\mathbb{P}^{\theta}$ (that on $\mathbb{Z}^{\mathbb{N}}$ ), denoted also $\mathbb{P}^{\theta}$ when no confusion occurs, is called the annealed law of walk $\mathbf{X}$. We denote by $\mathbb{E}^{\theta}$ the corresponding expectation. Note that the first marginal of $\mathbb{P}^{\theta}$ is the law of the Markov chain $\boldsymbol{\omega}$, denoted by $\mathbf{P}^{\theta}$. 
For all $k \in \mathbb{Z}$, we let

$$
\tilde{\omega}_{k}=\frac{1-\omega_{k}}{\omega_{k}} .
$$

In the case of an i.i.d. environment $\boldsymbol{\omega}$, [38] gives the classification of $\mathbf{X}$ between transient or recurrent cases according to whether $\mathbf{E}^{\theta}\left(\log \tilde{\omega}_{0}\right)$ is different or not from zero. For stationary ergodic environments, which is the case here, [2] establishes that this characterization remains valid. Thus, if $\mathbf{E}^{\theta}\left(\log \tilde{\omega}_{0}\right)<0$, then the walk is transient to the right, namely

$$
\lim _{t \rightarrow \infty} X_{t}=+\infty, \quad \mathbb{P}^{\theta}-\text { a.s. }
$$

Let $T_{n}$ be the first hitting time of the positive integer $n$,

$$
T_{n}=\inf \left\{t \in \mathbb{N}: X_{t}=n\right\}
$$

and define

$$
R=\left(1+\tilde{\omega}_{1}+\tilde{\omega}_{1} \tilde{\omega}_{2}+\ldots\right) .
$$

Theorem 4.1 in [2] shows that if the environment satisfies the condition

$$
\mathbf{E}^{\theta}(R)<+\infty,
$$

then the speed of the walk is strictly positive. Namely, $\mathbb{P}^{\theta}$-almost surely, the ratio $T_{n} / n$ converges to a finite limit as $n$ increases. Thus (2.3) gives the so-called ballistic condition on the random walk with Markov environment. Note that in the i.i.d. case, this condition reduces to $\mathbf{E}^{\theta}\left(\tilde{\omega}_{0}\right)<1$. Moreover, in the non independent case, when the distribution of the environment is uniquely ergodic, namely $\boldsymbol{\omega}$ is not i.i.d. and admits a unique invariant distribution, [2] establishes that transience (namely $\left.\mathbf{E}^{\theta}\left(\log \tilde{\omega}_{0}\right)<0\right)$ automatically implies ballistic regime (see Lem. 6.1 in [2]). Since in our context we assume that the Markov environment $\boldsymbol{\omega}$ admits a unique invariant distribution, the ballistic assumption thus reduces to

$$
\left\{\begin{array}{l}
\mathbf{E}^{\theta}\left(\tilde{\omega}_{0}\right)<1 \quad \text { if } \boldsymbol{\omega} \text { i.i.d, } \\
\mathbf{E}^{\theta}\left(\log \tilde{\omega}_{0}\right)<0 \quad \text { if } \boldsymbol{\omega} \text { non independent. }
\end{array}\right.
$$

In the following, we consider a transient to the right ballistic process $\mathbf{X}$.

\subsection{Problem and motivation}

We consider a finite trajectory $\mathbf{X}_{n}=\left(X_{t}\right)_{t \leq T_{n}}$ from the process $\mathbf{X}$, stopped at the first hitting time of a positive integer $n \geq 1$. The apparently more general case of a sequence $\left(X_{1}, \ldots, X_{n}\right)$ of observations is discussed in Remark 3.3. We assume that this sequence of observations is generated under $\mathbb{P}^{\theta^{\star}}:=\mathbb{P}^{\star}$ for a true parameter value $\theta^{\star}$ belonging to the interior $\Theta_{\Theta}$ of $\Theta$. Our goal is to estimate this parameter value $\theta^{\star}$ from the sequence of observations $\mathbf{X}_{n}$ using a maximum likelihood approach. To motivate the following developments, we will first explain why we can not directly rely on the likelihood of these observations. Indeed, let $\mathcal{V}_{n}$ be the set of sites $x \in \mathbb{Z}$ visited by the process up to time $T_{n}$, namely

$$
\mathcal{V}_{n}=\left\{x \in \mathbb{Z} ; \exists 0 \leq s \leq T_{n}, X_{s}=x\right\} .
$$

Under the assumption of a transient (to the right) process, the random set $\mathcal{V}_{n}$ is equal to $\llbracket \xi_{n}, n \rrbracket$, where $\xi_{n} \in \mathbb{Z}^{-}$ and $\llbracket a, b \rrbracket$ denotes the set of integers between $a$ and $b$ for any $a \leq b$ in $\mathbb{Z}$. Here, $\xi_{n}$ is the smallest integer value visited by the process $\mathbf{X}_{n}$. We also introduce $\boldsymbol{\omega}\left(\mathbf{X}_{n}\right):=\left(\omega_{\xi_{n}}, \ldots, \omega_{n}\right)$ which is the random environment restricted to the set of sites visited by $\mathbf{X}_{n}$. Now, the likelihood of $\mathbf{X}_{n}$ is given by the following expression

$$
\begin{aligned}
\mathbb{P}^{\theta}\left(\mathbf{X}_{n}\right) & =\int_{S} \ldots \int_{S} \mathbb{P}^{\theta}\left(\boldsymbol{\omega}\left(\mathbf{X}_{n}\right)=\left(a_{\xi_{n}}, \ldots, a_{n}\right), \mathbf{X}_{n}\right) \mathrm{d} a_{\xi_{n}} \ldots \mathrm{d} a_{n} \\
& =\int_{S} \ldots \int_{S} \mu_{\theta}\left(a_{\xi_{n}}\right) \prod_{i=\xi_{n}}^{n-1} q_{\theta}\left(a_{i}, a_{i+1}\right) \prod_{s=0}^{T_{n}-1} p_{a_{X_{s}}}\left(X_{s}, X_{s+1}\right) \mathrm{d} a_{\xi_{n}} \ldots \mathrm{d} a_{n} .
\end{aligned}
$$


Computing the likelihood from the above expression would require to compute $\left|\mathcal{V}_{n}\right|$ integral terms (where $|\cdot|$ denotes cardinality). As $\left|\mathcal{V}_{n}\right| \geq n$, this means that using a discretization method over $N$ points for each integral (or letting $N$ be the cardinality of $S$ ) would result in summing over at least $N^{n}$ different terms. This is unfeasible but for small values of $n$. Moreover, the above expression is not well suited for studying the convergence properties of this likelihood. Following [18], instead of focusing on the observed process $\mathbf{X}_{n}$, we will rather consider the underlying sequence $L_{n}^{n}, L_{n-1}^{n}, \ldots, L_{0}^{n}$ of the number of left steps of the process $\mathbf{X}_{n}$ at the sequence of sites $(n, n-1, \ldots, 0)$ and construct our estimator from this latter sequence. Though we do not need it, note that it is argued in [18] that the latter is in fact a sufficient statistic (at least asymptotically) for the parameter $\theta$. In the next section, we show that in the case of a Markov environment, this process exhibits a hidden Markov structure. Moreover for transient RWRE, this process is recurrent, allowing us to study the convergence properties of MLE.

\subsection{The underlying hidden Markov chain}

We define the sequence of left steps at each visited site from the (positive part of the) trajectory $\mathbf{X}_{n}$ as follows. Let

$$
L_{x}^{n}:=\sum_{s=0}^{T_{n}-1} \mathbb{1}\left\{X_{s}=x ; X_{s+1}=x-1\right\}, \quad \forall x \in\{0, \ldots, n\} .
$$

It is observed by [29] in the case of an i.i.d. random environment that the sequence $\left(L_{n}^{n}, L_{n-1}^{n}, \ldots, L_{0}^{n}\right)$ is distributed as a branching process with immigration in a random environment (BPIRE). We will first show that this remains true in the case of a Markov environment. To this aim, let us introduce the time reversed environment $\breve{\boldsymbol{\omega}}=\left(\breve{\omega}_{x}\right)_{x \in \mathbb{Z}}$ defined by $\breve{\omega}_{x}=\omega_{-x}$ for all $x \in \mathbb{Z}$. It is a Markov chain on $S$ with stationary density $\mu_{\theta}$ and transition $\breve{q}_{\theta}$ defined by

$$
\forall a, b \in S, \quad \breve{q}_{\theta}(a, b)=\frac{\mu_{\theta}(b) q_{\theta}(b, a)}{\mu_{\theta}(a)} .
$$

Now we recursively define a sequence of random variables $\left(Z_{k}\right)_{k \geq 0}$ with $Z_{0}=0$ and

$$
\forall k \geq 0, \quad Z_{k+1}=\sum_{i=0}^{Z_{k}} \xi_{k+1, i}
$$

where for all $k \geq 1$, the random variables $\left(\xi_{k, i}\right)_{i \in \mathbb{N}}$, are defined on the same probability space as previously, are independent and their conditional distribution, given the environment $\breve{\omega}$ is

$$
\forall m \in \mathbb{N}, \quad P_{\breve{\omega}}\left(\xi_{k, i}=m\right)=\left(1-\breve{\omega}_{k}\right)^{m} \breve{\omega}_{k} .
$$

Here, $P_{\breve{\omega}}$ is defined similarly as $P_{\boldsymbol{\omega}}$ for the environment $\breve{\boldsymbol{\omega}}$ replacing $\boldsymbol{\omega}$. Then, conditionally on $\breve{\boldsymbol{\omega}}$, the sequence $\left(Z_{k}\right)_{k \in \mathbb{N}}$ is an inhomogeneous branching process with immigration, with identical offspring and immigration law, given by a geometric distribution (whose parameter depends on the random environment $\breve{\boldsymbol{\omega}}$ ). Moreover, it is easily seen that the annealed distribution of the sequence $\left(L_{n}^{n}, L_{n-1}^{n}, \ldots, L_{0}^{n}\right)$ and that of $\left(Z_{0}, Z_{1}, \ldots, Z_{n}\right)$ are the same.

Lemma 2.1. For any fixed integer $n \geq 1$, the sequence of left steps $\left(L_{n}^{n}, L_{n-1}^{n}, \ldots, L_{0}^{n}\right)$ has same distribution as $\left(Z_{0}, Z_{1}, \ldots, Z_{n}\right)$ under $\mathbb{P}^{\theta}$.

Proof. For any fixed integer $n \geq 1$, let $\overline{\boldsymbol{\omega}}^{n}:=\left(\omega_{n}, \omega_{n-1}, \ldots, \omega_{0}, \omega_{-1}, \ldots\right)$ denote the time reversed environment starting at $\omega_{n}$. Let also $P_{\overline{\boldsymbol{\omega}}^{n}}$ be defined similarly as $P_{\boldsymbol{\omega}}$ for the environment $\overline{\boldsymbol{\omega}}^{n}$ replacing $\boldsymbol{\omega}$. Then it is known that for any sequence $\left(z_{0}, \ldots, z_{n}\right) \in \mathbb{N}^{n+1}$, we have the equality

$$
P_{\boldsymbol{\omega}}\left(\left(L_{n}^{n}, L_{n-1}^{n}, \ldots, L_{0}^{n}\right)=\left(z_{0}, \ldots, z_{n}\right)\right)=P_{\overline{\boldsymbol{\omega}}^{n}}\left(\left(Z_{0}, \ldots, Z_{n}\right)=\left(z_{0}, \ldots, z_{n}\right)\right)
$$


(see for instance Sect. 4.1 in [18]). Now the right-hand side $P_{\overline{\boldsymbol{\omega}}^{n}}\left(\left(Z_{0}, \ldots, Z_{n}\right)=\left(z_{0}, \ldots, z_{n}\right)\right)$ only depends on the environment $\overline{\boldsymbol{\omega}}^{n}$ through the first $(n+1)$ variables $\left(\omega_{n}, \omega_{n-1}, \ldots, \omega_{0}\right)$ whose distribution under $\mathbf{P}^{\theta}$ is the same as $\left(\breve{\omega}_{0}, \breve{\omega}_{1}, \ldots, \breve{\omega}_{n}\right)$. As a consequence, using definition (2.1) of distribution $\mathbb{P}^{\theta}$ we obtain the result.

When the environment $\boldsymbol{\omega}$ is composed of i.i.d. random variables, the resulting sequence $\left(Z_{k}\right)_{k \geq 0}$ is a homogeneous Markov chain under $\mathbb{P}^{\theta}$ (see e.g. [18]). Now, when the environment $\boldsymbol{\omega}$ is itself a Markov chain, we observe that $\left(Z_{k}\right)_{k>0}$ is distributed as a hidden Markov chain, or more precisely as the second marginal of a first order autoregressive process with Markov regime [22], where the latent sequence is given by $\breve{\omega}$. We state this result as a lemma (namely Lem. 2.2 below) even though its proof is obvious and thus omitted. Let us recall that a first order autoregressive process with Markov regime (or Markov-switching autoregression) is a bivariate process $\left\{\left(\breve{\omega}_{k}, Z_{k}\right)\right\}_{k \geq 0}$ such that $\breve{\boldsymbol{\omega}}=\left(\breve{\omega}_{k}\right)_{k \geq 0}$ is a Markov chain and conditionally on $\breve{\boldsymbol{\omega}}$, the sequence $\left(Z_{k}\right)_{k \geq 0}$ is an inhomogeneous Markov chain whose transition from $Z_{k-1}$ to $Z_{k}$ only depends on $Z_{k-1}$ and $\breve{\omega}_{k}$.

For any $a \in S$ and $(x, y) \in \mathbb{N}^{2}$, denote

$$
g_{a}(x, y)=\left(\begin{array}{c}
x+y \\
x
\end{array}\right) a^{x+1}(1-a)^{y}
$$

and let $\delta_{x}$ be the Dirac measure at $x$. Let us recall that the process $\left(Z_{k}\right)_{k \geq 0}$ is defined through (2.6) and (2.7).

Lemma 2.2. Under $\mathbb{P}^{\theta}$, the process $\left\{\left(\breve{\omega}_{k}, Z_{k}\right)\right\}_{k \geq 0}$ is a first-order autoregressive process with Markov regime. The first component $\breve{\boldsymbol{\omega}}$ is an homogenous Markov chain with transition kernel density $\breve{q}_{\theta}$ and initial distribution $\mu_{\theta}$. Conditionally on $\breve{\boldsymbol{\omega}}$, the process $\left(Z_{k}\right)_{k \in \mathbb{N}}$, is an inhomogeneous Markov chain, starting from $Z_{0}=0$ and with transitions

$$
\forall(x, y) \in \mathbb{N}^{2}, \forall k \in \mathbb{N} \quad P_{\breve{\omega}}\left(Z_{k+1}=y \mid Z_{k}=x\right)=g_{\breve{\omega}_{k+1}}(x, y) .
$$

As a consequence, $\left\{\left(\breve{\omega}_{k}, Z_{k}\right)\right\}_{k \geq 0}$ is a Markov chain with state space $S \times \mathbb{N}$, starting from $\mu_{\theta} \otimes \delta_{0}$ and with transition kernel density $\Pi_{\theta}$ defined for all $(a, b, x, y) \in S^{2} \times \mathbb{N}^{2}$ by

$$
\Pi_{\theta}((a, x),(b, y))=\breve{q}_{\theta}(a, b) g_{b}(x, y) .
$$

Remark 2.3. The conditional autoregressive part of the distribution, given by (2.8), is usually referred to as emission distribution. Note that in our framework, this law does not depend on the parameter $\theta$.

Under $\mathbb{P}^{\theta}$, the process $(\breve{\boldsymbol{\omega}}, Z)$ has initial distribution $\mu_{\theta} \otimes \delta_{0}$. In the sequel, we also need $(\breve{\boldsymbol{\omega}}, Z)$ as well as the chain $\left(\omega_{k}\right)_{k>0}$ starting from any initial distribution. For any probability $\nu$ on $\mathcal{B}(S \times \mathbb{N})$, denote $\mathbb{P}_{\nu}^{\theta}$ the law of $(\breve{\boldsymbol{\omega}}, Z)$ starting from $\left(\omega_{0}, Z_{0}\right) \sim \nu$ (note that $\left.\breve{\omega}_{0}=\omega_{0}\right)$. Denote $\mathbb{E}_{\nu}^{\theta}$ the corresponding expectation. In particular, for $(a, x) \in S \times \mathbb{N}$, we let $\mathbb{P}_{(a, x)}^{\theta}$ and $\mathbb{E}_{(a, x)}^{\theta}$ be the probability and expectation if $\left(\omega_{0}, Z_{0}\right)=(a, x)$. Moreover, when only the first component is concerned and when no confusion occurs, if the chain $\breve{\boldsymbol{\omega}}$ or $\boldsymbol{\omega}$ starts from its stationary distribution $\mu_{\theta}$, we still denote this marginal law by $\mathbf{P}^{\theta}$ and the corresponding expectation by $\mathbf{E}^{\theta}$. If $\breve{\boldsymbol{\omega}}$ or $\boldsymbol{\omega}$ start from another initial law, for example $\omega_{0}=a$, we denote their law by $\mathbf{P}_{a}^{\theta}$ and corresponding expectation $\mathbf{E}_{a}^{\theta}$. For $n \in \mathbb{N}$, we let $\mathcal{F}_{n}=\sigma\left\{\omega_{k}, k=0, \ldots, n\right\}$ (resp. $\breve{\mathcal{F}}_{n}=\sigma\left\{\breve{\omega}_{k}, k=0, \ldots, n\right\}$ ) be the $\sigma$-field induced by the $(n+1)$ first random variables of the environment (resp. of the time reversed environment). Moreover, we denote by $\boldsymbol{\omega}^{n}=\left(\omega_{n}, \omega_{n+1}, \ldots\right)$ and $\breve{\boldsymbol{\omega}}^{n}=\left(\breve{\omega}_{n}, \breve{\omega}_{n+1}, \ldots\right)$ the shifted sequences. The family of shift operators $\left(\tau^{n}\right)_{n \geq 1}$ where $\tau^{n}: \Omega \rightarrow \Omega$ is defined by

$$
\forall \boldsymbol{\omega}, \breve{\boldsymbol{\omega}} \in \Omega, \quad \tau^{n}(\boldsymbol{\omega})=\boldsymbol{\omega}^{n} \text { and } \tau^{n}(\breve{\boldsymbol{\omega}})=\breve{\boldsymbol{\omega}}^{n} .
$$

In Section 5, we show that under the ballistic assumption, the bivariate kernel $\Pi_{\theta}((a, x),(b, y))$ is positive Harris recurrent and admits a unique invariant distribution with density $\pi_{\theta}$, for which we give an explicit formula (see Prop. 5.1). In the following, we let $\overline{\mathbb{P}}^{\theta}$ and $\overline{\mathbb{E}}^{\theta}$ be the probability and expectation induced when considering the chain $\left\{\left(\breve{\omega}_{k}, Z_{k}\right)\right\}_{k \geq 0}$ under its stationary distribution $\pi_{\theta}$. 


\section{Assumptions And Results}

\subsection{Estimator construction}

Recall that our aim is to infer the unknown parameter $\theta^{\star} \in \Theta$, using the observation of a finite trajectory $\mathbf{X}_{n}$ up to the first hitting time $T_{n}$ of site $n$. The observed trajectory is transformed into the sequence $L_{n}^{n}, L_{n-1}^{n}, \ldots, L_{0}^{n}$ of the number of left steps of the process $\mathbf{X}_{n}$ at the sequence of sites $(n, n-1, \ldots, 0)$. This trajectory is generated under the law $\mathbb{P}^{\star}$ (recall that $\mathbb{P}^{\star}$ is a shorthand notation of $\mathbb{P}^{\theta^{*}}$ ). Due to the equality in law given by Lemma 2.1 , we can consider that we observe a sequence of random variables $\left(Z_{0}, \ldots, Z_{n}\right)$ which is the second component of an autoregressive process with Markov regime described in Lemma 2.2. Thus under $\mathbb{P}^{\star}$, the law of the MLE of these observations is the same than the law of MLE built from $\left(Z_{0}, \ldots, Z_{n}\right)$.

As a consequence, we can now rely on a set of well established techniques developed in the context of autoregressive processes with Markov regime, both for computing efficiently the likelihood and for establishing its asymptotic properties. Following [22], we define a conditional log-likelihood, conditioned on an initial state of the environment $\breve{\omega}_{0}=a_{0} \in S$. The reason for doing so is that the stationary distribution of $\left.\left\{\breve{\omega}_{k}, Z_{k}\right)\right\}_{k \geq 0}$ and hence the true likelihood, is typically infeasible to compute.

Definition 3.1. Fix some $a_{0} \in S$ and consider the conditional log-likelihood of the observations defined as

$$
\ell_{n}\left(\theta, a_{0}\right):=\log \mathbb{P}_{\left(a_{0}, 0\right)}^{\theta}\left(Z_{1}, \ldots, Z_{n}\right)=\log \int_{S^{n}} \prod_{i=1}^{n} \breve{q}_{\theta}\left(a_{i-1}, a_{i}\right) g_{a_{i}}\left(Z_{i-1}, Z_{i}\right) \mathrm{d} a_{i} .
$$

Note that the above expression of the (conditional) log-likelihood shares the computational problems mentioned for expression (2.5). However, in the present context of autoregressive processes with Markov regime, efficient computation of this expression is possible. The key ingredient for this computation (that also serves to study the convergence properties of $\ell_{n}$ ) is to rely on the following additive form

$$
\begin{aligned}
\ell_{n}\left(\theta, a_{0}\right) & =\sum_{k=1}^{n} \log \mathbb{P}_{\left(a_{0}, 0\right)}^{\theta}\left(Z_{k} \mid Z_{0}, \ldots, Z_{k-1}\right) \\
& =\sum_{k=1}^{n} \log \left(\iint_{S^{2}} g_{b}\left(Z_{k-1}, Z_{k}\right) \breve{q}_{\theta}(a, b) \mathbb{P}_{\left(a_{0}, 0\right)}^{\theta}\left(\breve{\omega}_{k-1}=a \mid Z_{0}^{k-1}\right) \mathrm{d} a \mathrm{~d} b\right),
\end{aligned}
$$

where $Z_{s}^{t}$ denotes $Z_{s}, Z_{s+1}, \ldots, Z_{t}$ for any integers $s \leq t$. We further develop this point in Section 4.1 and also refer to [22] for more details.

Definition 3.2. The estimator $\hat{\theta}_{n}$ is defined as a measurable choice

$$
\hat{\theta}_{n} \in \underset{\theta \in \Theta}{\operatorname{Argmax}} \ell_{n}\left(\theta, a_{0}\right) .
$$

Note that we omit the dependence of $\hat{\theta}_{n}$ on the initial state $a_{0}$ of the environment.

Remark 3.3. When considering a size- $n$ sample $X_{1}, \ldots X_{n}$ instead of a trajectory stopped at random time $T_{n}$, we may consider $m:=m(n)=\max _{1 \leq i \leq n} X_{i}$ and restrict our attention to the sub-sample $X_{1}, \ldots, X_{T_{m}}$. As we consider a transient random walk, $m(n)$ increases to infinity with $n$. Consistency with respect to $n$ or $m(n)$ is equivalent. Now considering the rates, note that in the ballistic case we can easily obtain that $m(n) \sim c n$ for some $c>0$ so that rates of convergence as well as efficiency issues with respect to $m(n)$ or $n$ are the same. Note that information about $T_{m}$ has to be extracted first and then the data may be reduced to the sequence of left steps without loosing information. 


\subsection{Assumptions and results}

Recall that $q_{\theta}$ and $\mu_{\theta}$ are respectively the transition and the invariant probability densities of the environment Markov chain $\boldsymbol{\omega}$ with values in $S$, while $\breve{q}_{\theta}$ and $\mu_{\theta}$ are the same quantities for the time reversed chain $\breve{\boldsymbol{\omega}}$. Moreover, $S$ is a closed subset of $(0,1)$ so that we can assume that there exists some $\varepsilon \in(0,1)$ such that

$$
S \subseteq[\varepsilon ; 1-\varepsilon] .
$$

The above assumption is known as the uniform ellipticity condition.

We also recall that the random variable $R$ is defined by (2.2).

Assumption 1 (Ballistic case). For any $\theta \in \Theta$, Inequality (2.4) is satisfied.

Assumption 2. There exist some constants $0<\sigma_{-}, \sigma_{+}<+\infty$ such that

$$
\sigma_{-} \leq \inf _{\theta \in \Theta} \inf _{a, b \in S} q_{\theta}(a, b) \leq \sup _{\theta \in \Theta} \sup _{a, b \in S} q_{\theta}(a, b) \leq \sigma_{+} .
$$

Note that it easily follows from this assumption that the stationary density $\mu_{\theta}$ also satisfies

$$
\sigma_{-} \leq \inf _{\theta \in \Theta} \inf _{a \in S} \mu_{\theta}(a) \leq \sup _{\theta \in \Theta} \sup _{a \in S} \mu_{\theta}(a) \leq \sigma_{+} .
$$

Moreover, we also get that the time reversed transition density $\breve{q}_{\theta}$ satisfies

$$
\frac{\sigma_{-}^{2}}{\sigma_{+}} \leq \inf _{\theta \in \Theta} \inf _{a, b \in S} \breve{q}_{\theta}(a, b) \leq \sup _{\theta \in \Theta} \sup _{a, b \in S} \breve{q}_{\theta}(a, b) \leq \frac{\sigma_{+}^{2}}{\sigma_{-}} .
$$

Assumptions 1 and 2 are used to establish that the bivariate process $\left\{\left(\breve{\omega}_{k}, Z_{k}\right)\right\}_{k \geq 0}$ is positive Harris recurrent. Note in particular that the weakest assumptions currently ensuring consistency of the MLE in the HMM setting contain positive Harris recurrence of the hidden chain [21] and Assumption 2 is further required in the less simple case of an autoregressive model with Markov regime [22]. The lower bound in Assumption 2 may be restrictive in a general HMM setting as it prevents the support $S$ from being unbounded. However here we have $S \subseteq(0,1)$ and thus Assumption 2 is satisfied in many examples (see Sect. 4).

Next assumption is classical from a statistical perspective and requires smoothness of the underlying model.

Assumption 3 (Regularity condition). For all $(a, b) \in S^{2}$, the map $\theta \mapsto q_{\theta}(a, b)$ is continuous.

In order to ensure identifiability of the model, we naturally require identifiability of the parameter from the distribution of the environment.

Assumption 4 (Identifiability condition).

$$
\forall \theta, \theta^{\prime} \in \Theta, \quad \theta=\theta^{\prime} \Longleftrightarrow q_{\theta}=q_{\theta^{\prime}} .
$$

Theorem 3.4. Under Assumptions 1 to 4 , the maximum likelihood estimator $\hat{\theta}_{n}$ converges $\mathbb{P}^{\star}$-almost surely to the true parameter value $\theta^{\star}$ as $n$ tends to infinity.

We now introduce the conditions that will ensure asymptotic normality of $\hat{\theta}_{n}$ under $\mathbb{P}^{\star}$. In the following, for any function $\varphi: \Theta \mapsto \mathbb{R}$, we let $\partial_{\theta} \varphi$ and $\partial_{\theta}^{2} \varphi$ denote gradient vector and Hessian matrix, respectively. Moreover, $\|\cdot\|$ is the uniform norm (of a vector or a matrix). Again, next condition is classical and requires regularity of the mapping underlying the statistical model. 
Assumption 5. For all $(a, b) \in S^{2}$, the map $\theta \mapsto q_{\theta}(a, b)$ is twice continuously differentiable on $\Theta$. Moreover,

$$
\begin{array}{r}
\sup _{\theta \in \Theta} \sup _{a, b \in S}\left\|\partial_{\theta} \log q_{\theta}(a, b)\right\|<+\infty, \quad \sup _{\theta \in \sup _{a, b \in S}}\left\|\partial_{\theta}^{2} \log q_{\theta}(a, b)\right\|<+\infty, \\
\quad \sup _{\theta \in \Theta} \sup _{a \in S}\left\|\partial_{\theta} \log \mu_{\theta}(a)\right\|<+\infty \text { and } \sup _{\theta \in \Theta} \sup _{a \in S}\left\|\partial_{\theta}^{2} \log \mu_{\theta}(a)\right\|<+\infty .
\end{array}
$$

Following the notation from Section 6.1 in [22], we now introduce the asymptotic Fisher information matrix. We start by extending the chain $\left\{\left(\breve{\omega}_{k}, Z_{k}\right)\right\}_{k \in \mathbb{N}}$ with indexes in $\mathbb{N}$ to a stationary Markov chain $\left\{\left(\breve{\omega}_{k}, Z_{k}\right)\right\}_{k \in \mathbb{Z}}$ indexed by $\mathbb{Z}$. Let us recall that $\overline{\mathbb{P}}^{\theta}$ and $\overline{\mathbb{E}}^{\theta}$ respectively denote probability and expectation under the stationary distribution $\pi_{\theta}$ of the chain $\left\{\left(\breve{\omega}_{k}, Z_{k}\right)\right\}_{k>0}$. For any $k \geq 1, m \geq 0$, we let

$$
\Delta_{k, m}(\theta)=\overline{\mathbb{E}}^{\theta}\left(\sum_{i=-m+1}^{k} \partial_{\theta} \log \breve{q}_{\theta}\left(\breve{\omega}_{i-1}, \breve{\omega}_{i}\right) \mid Z_{-m}^{k}\right)-\overline{\mathbb{E}}^{\theta}\left(\sum_{i=-m+1}^{k-1} \partial_{\theta} \log \breve{q}_{\theta}\left(\breve{\omega}_{i-1}, \breve{\omega}_{i}\right) \mid Z_{-m}^{k-1}\right) .
$$

Note that this expression derives from Fisher identity stated in [32]. Indeed, under general assumptions, the score function equals the conditional expectation of the complete score, given the observed data. As the emission distribution $g$ does not depend on the parameter $\theta$, the complete score reduces to a sum of terms involving $\breve{q}_{\theta}$ only.

Lemma 10 in [22] establishes that for any $k \geq 1$, the sequence $\left(\Delta_{k, m}\left(\theta^{\star}\right)\right)_{m>0}$ converges in $\mathbb{L}^{2}\left(\overline{\mathbb{P}}^{\star}\right)$ to some limit $\Delta_{k, \infty}\left(\theta^{\star}\right)$. From this quantity, we may define

$$
I\left(\theta^{\star}\right)=\overline{\mathbb{E}}^{\star}\left(\Delta_{0, \infty}\left(\theta^{\star}\right)^{\top} \Delta_{0, \infty}\left(\theta^{\star}\right)\right),
$$

where by convention $\Delta_{0, \infty}$ is a row vector and $u^{\top}$ is the transpose vector of $u$. Then, $I\left(\theta^{\star}\right)$ is the Fisher information matrix of the model. We can now state the asymptotic normality result.

Theorem 3.5. Under Assumptions 1 to 5, if the asymptotic Fisher information matrix $I\left(\theta^{\star}\right)$ defined by (3.5) is invertible, we have that

$$
n^{-1 / 2}\left(\hat{\theta}_{n}-\theta^{\star}\right) \underset{n \rightarrow+\infty}{\longrightarrow} \mathcal{N}\left(0, I\left(\theta^{\star}\right)^{-1}\right), \quad \mathbb{P}^{\star} \text {-weakly }
$$

Note that the definition of $I\left(\theta^{\star}\right)$ is not constructive. In particular, asymptotic normality of the MLE requires that $I\left(\theta^{\star}\right)$ is invertible but this may not be ensured through more explicit conditions on the original process. However, the Fisher information may be approximated through the Hessian of the log-likelihood. Indeed, Theorem 3 in [22] states that the normalized Hessian of the log-likelihood converges to $-I\left(\theta^{\star}\right)$ under stationary distribution $\overline{\mathbb{P}}^{\star}$. Moreover, this result is generalized to obtain convergence under non stationary distribution $\mathbb{P}^{\star}$ (see the proof of Thm. 6 in that reference). Thus we have

$$
\frac{1}{n} \partial_{\theta}^{2} \ell_{n}\left(\hat{\theta}_{n}\right) \underset{n \rightarrow+\infty}{\longrightarrow}-I\left(\theta^{\star}\right), \quad \mathbb{P}^{\star}-\text { a.s. }
$$

In practice, this may be used to approximate the asymptotic variance of the estimator $\hat{\theta}_{n}$, as illustrated in Section 4.3.

\section{Illustration: ExAmPles AND SIMUlations}

\subsection{Computation of the likelihood}

The computation of the log-likelihood relies on the following set of equations. As already noted, we have

$$
\begin{aligned}
\ell_{n}(\theta, a) & =\sum_{k=1}^{n} \log \mathbb{P}_{(a, 0)}^{\theta}\left(Z_{k} \mid Z_{0}^{k-1}\right), \\
& =\sum_{k=1}^{n} \log \left(\iint_{S^{2}} g_{b^{\prime}}\left(Z_{k-1}, Z_{k}\right) \breve{q}_{\theta}\left(b, b^{\prime}\right) \mathbb{P}_{(a, 0)}^{\theta}\left(\breve{\omega}_{k-1}=b \mid Z_{0}^{k-1}\right) \mathrm{d} b \mathrm{~d} b^{\prime}\right) .
\end{aligned}
$$


In this expression, the quantity

$$
F_{k}^{\theta, a}(\cdot)=\mathbb{P}_{(a, 0)}^{\theta}\left(\breve{\omega}_{k}=\cdot \mid Z_{0}^{k}\right),
$$

is the called the prediction filter. It is a probability distribution on $S$ and it is computed through recurrence relations. Indeed, we have

$$
\left\{\begin{array}{l}
F_{0}^{\theta, a}=\delta_{a}, \\
F_{k+1}^{\theta, a}\left(b^{\prime}\right) \propto g_{b^{\prime}}\left(Z_{k}, Z_{k+1}\right) \int_{S} \breve{q}_{\theta}\left(b, b^{\prime}\right) F_{k}^{\theta, a}(b) \mathrm{d} b, \quad k \geq 0, b^{\prime} \in S,
\end{array}\right.
$$

where $\propto$ means proportional to (up to a normalizing constant).

When $S$ is discrete, the integral terms over $S$ reduce to sums and computing the prediction filter recursively enables to compute the log-likelihood of the observations, and then the MLE. We illustrate these computations in the case of Example 2 below as well as in Section 4.3. When $S$ is continuous, approximation methods are required, e.g. particle filters or Monte Carlo expectation-maximisation (em) algorithms. We refer to Section 8 in [22] for more details.

Note that in any case, optimisation of the log-likelihood is either done through em algorithm $[9,19]$ or by direct optimisation procedures, as there is no analytical expression for its maximiser. Thus, the computation of the gradient of this log-likelihood is often used (e.g. in descent gradient optimisation methods). As soon as we can differentiate under the integral sign (which is valid under Assumption 5), the gradient function $\partial_{\theta} \ell_{n}(\theta, a)$ writes

$$
\begin{aligned}
\partial_{\theta} \ell_{n}(\theta, a)= & \left(\iint_{S^{2}} g_{b^{\prime}}\left(Z_{k-1}, Z_{k}\right) \breve{q}_{\theta}\left(b, b^{\prime}\right) F_{k-1}^{\theta, a}(b) \mathrm{d} b \mathrm{~d} b^{\prime}\right)^{-1} \\
& \times \sum_{k=1}^{n}\left(\iint_{S^{2}} g_{b^{\prime}}\left(Z_{k-1}, Z_{k}\right)\left[\partial_{\theta} \breve{q}_{\theta}\left(b, b^{\prime}\right) F_{k-1}^{\theta, a}(b)+\breve{q}_{\theta}\left(b, b^{\prime}\right) \partial_{\theta} F_{k-1}^{\theta, a}(b)\right] \mathrm{d} b \mathrm{~d} b^{\prime}\right) .
\end{aligned}
$$

Note that the gradient of the prediction filter $\partial_{\theta} F_{k-1}^{\theta, a}$ may be obtained through recurrence relations similar to (4.3). However, these relations are more involved since the normalizing constant in (4.3) depends on $\theta$ and can not be neglected.

To conclude this section, we mention that computing the Hessian of the log-likelihood can be done in a similar way.

\subsection{Examples}

In this section, we provide some examples of environments $\boldsymbol{\omega}$ and check the assumptions needed for consistency and asymptotic normality of the MLE.

Example 1 (Simple i.i.d. environment on two values). Let $q_{\theta}(a, b)=\mu_{\theta}(b)$ and $\mu_{\theta}(\cdot)=\mu_{p}(\cdot)=p \delta_{a_{1}}(\cdot)+(1-$ $p) \delta_{a_{2}}(\cdot)$ with known values $a_{1}, a_{2} \in(0,1)$ and unknown parameter $p \in[\gamma, 1-\gamma] \subseteq(0,1)$.

The support of the environment is reduced to $S=\left\{a_{1}, a_{2}\right\}$. Moreover, we assume that $a_{1}, a_{2}$ and $\Theta$ are such that the process is transient to the right and ballistic. In the i.i.d. case, the ballistic assumption (that also implies transience) reduces to $\mathbf{E}^{\theta}\left(\tilde{\omega}_{0}\right)<1$ and thus to

$$
p \frac{1-a_{1}}{a_{1}}+(1-p) \frac{1-a_{2}}{a_{2}}<1 .
$$

The log-likelihood of the observations has a very simple form in this setup

$$
\ell_{n}(p)=\sum_{k=1}^{n} \log \left[p a_{1}^{Z_{k-1}+1}\left(1-a_{1}\right)^{Z_{k}}+(1-p) a_{2}^{Z_{k-1}+1}\left(1-a_{2}\right)^{Z_{k}}\right]
$$


and its maximiser $\hat{\theta}_{n}=\hat{p}_{n}$ is obtained through numerical optimisation. We refer to [18,25] for previous results obtained in this setup.

Assumptions 2 and 4 are satisfied as soon as $\Theta \subseteq[\gamma, 1-\gamma]$ and $a_{1} \neq a_{2}$, respectively. Moreover, Assumptions 3 and 5 are automatically satisfied. Indeed, for any $p \in \Theta$ and any $a \in S$, we have

$$
\begin{aligned}
\left|\partial_{p} \log \mu_{p}(a)\right| & =\frac{1}{p \mathbb{1}\left\{a=a_{1}\right\}+(1-p) \mathbb{1}\left\{a=a_{2}\right\}}, \\
\left|\partial_{p}^{2} \log \mu_{p}(a)\right| & =\frac{1}{p^{2} \mathbb{1}\left\{a=a_{1}\right\}+(1-p)^{2} \mathbb{1}\left\{a=a_{2}\right\}} .
\end{aligned}
$$

As a consequence, Theorems 3.4 and 3.5 are valid in this setup.

Example 2 (Finite Markov chain environment).

Let us assume that $S=\left\{a_{1}, a_{2}\right\}$ is fixed and known and the stationary Markov chain $\boldsymbol{\omega}$ is defined through its transition matrix

$$
Q_{\theta}=\left(\begin{array}{cc}
\alpha & 1-\alpha \\
1-\beta & \beta
\end{array}\right),
$$

where the parameter is $\theta=(\alpha, \beta) \in[\gamma, 1-\gamma]^{2}$ for some $\gamma>0$

Note that Assumption 2 is satisfied as soon as $\gamma>0$. The stationary measure of the Markov chain is given by

$$
\mu_{\theta}=\left(\frac{1-\beta}{2-\alpha-\beta}, \frac{1-\alpha}{2-\alpha-\beta}\right)
$$

This is automatically a reversible Markov chain so that $\breve{q}_{\theta}=q_{\theta}$. The transience condition writes

$$
(1-\beta) \log \left(\frac{1-a_{1}}{a_{1}}\right)+(1-\alpha) \log \left(\frac{1-a_{2}}{a_{2}}\right)<0 .
$$

Moreover, as soon as $\alpha \neq 1-\beta$ the sequence $\boldsymbol{\omega}$ is non independent and the existence of a unique stationary measure for $\boldsymbol{\omega}$ ensures the ballistic regime from transience assumption (Lem. 6.1 in [2]). Let us now consider the log-likelihood expression in this setup. As already explained, the key point for computing the log-likelihood in the setup of an autoregressive process with Markov regime is to rely on the following additive form

$$
\begin{aligned}
\ell_{n}\left(\theta, a_{1}\right) & =\sum_{k=1}^{n} \log \mathbb{P}^{\theta}\left(Z_{k} \mid Z_{0}^{k-1}, \omega_{0}=a_{1}\right) \\
& =\sum_{k=1}^{n} \log \left(\sum_{b, b^{\prime} \in S^{2}} g_{b^{\prime}}\left(Z_{k-1}, Z_{k}\right) q_{\theta}\left(b, b^{\prime}\right) F_{k-1}^{\theta, a_{1}}(b)\right),
\end{aligned}
$$

where $F_{k}^{\theta, a}$ is the prediction filter defined by (4.2) and we used $\breve{q}_{\theta}=q_{\theta}$. Relying on matrix notation, we let $F_{k}^{\theta, a}$ be the row vector $\left(F_{k}^{\theta, a}\left(a_{1}\right), F_{k}^{\theta, a}\left(a_{2}\right)\right)$ while $G_{k}$ is the row vector $\left(g_{a_{1}}\left(Z_{k-1}, Z_{k}\right), g_{a_{2}}\left(Z_{k-1}, Z_{k}\right)\right)$ and $u^{\top}$ the transpose vector of $u$. Then we obtain

$$
\ell_{n}\left(\theta, a_{1}\right)=\sum_{k=1}^{n} \log \left[F_{k-1}^{\theta, a_{1}} Q_{\theta} G_{k}^{\top}\right] .
$$

Moreover, the sequence of prediction filters $\left\{F_{k}^{\theta, a_{1}}\right\}_{0 \leq k \leq n-1}$ is obtained through the recurrence relations (4.3) that in our context, write as

$$
\left\{\begin{array}{l}
F_{0}^{\theta, a_{1}}=(1,0) \\
F_{k+1}^{\theta, a_{1}} \propto F_{k}^{\theta, a} Q_{\theta} \operatorname{Diag}\left(G_{k+1}\right) .
\end{array}\right.
$$


Now, the gradient function $\partial_{\theta} \ell_{n}(\theta, a)$ given by (4.4) satisfies the following equations

$$
\left\{\begin{array}{l}
\partial_{\alpha} \ell_{n}(\theta, a)=\sum_{k=1}^{n}\left[\left(\partial_{\alpha} F_{k-1}^{\theta, a_{1}} Q_{\theta}+F_{k-1}^{\theta, a_{1}} Q_{1}^{\prime}\right) G_{k}^{\top}\right]\left(F_{k-1}^{\theta, a_{1}} Q_{\theta} G_{k}^{\top}\right)^{-1}, \\
\partial_{\beta} \ell_{n}(\theta, a)=\sum_{k=1}^{n}\left[\left(\partial_{\beta} F_{k-1}^{\theta, a_{1}} Q_{\theta}+F_{k-1}^{\theta, a_{1}} Q_{2}^{\prime}\right) G_{k}^{\top}\right]\left(F_{k-1}^{\theta, a_{1}} Q_{\theta} G_{k}^{\top}\right)^{-1},
\end{array}\right.
$$

where $\partial_{i} F_{k-1}^{\theta, a_{1}}$ is the row vector with entries $\left(\partial_{i} F_{k-1}^{\theta, a_{1}}\left(a_{1}\right), \partial_{i} F_{k-1}^{\theta, a_{1}}\left(a_{2}\right)\right)$ and

$$
Q_{1}^{\prime}=\left(\begin{array}{cc}
1 & -1 \\
0 & 0
\end{array}\right), \quad Q_{2}^{\prime}=\left(\begin{array}{cc}
0 & 0 \\
-1 & 1
\end{array}\right)
$$

Let us denote by 1 the row vector $(1,1)$. In the current setup, the derivative of the prediction filter is obtained through $\partial_{\alpha} F_{0}^{\theta, a_{1}}=\partial_{\beta} F_{0}^{\theta, a_{1}}=(0,0)$ and for any $k \geq 0$,

$$
\begin{aligned}
\partial_{\alpha} F_{k+1}^{\theta, a_{1}}= & \left(F_{k}^{\theta, a_{1}} Q_{\theta} \operatorname{Diag}\left(G_{k+1}\right) \mathbf{1}^{\boldsymbol{\top}}\right)^{-1} \times\left(\partial_{\alpha} F_{k}^{\theta, a_{1}} Q_{\theta}+F_{k}^{\theta, a_{1}} Q_{1}^{\prime}\right) \operatorname{Diag}\left(G_{k+1}\right) \\
& -\frac{\left[\left(\partial_{\alpha} F_{k}^{\theta, a_{1}} Q_{\theta}+F_{k}^{\theta, a_{1}} Q_{1}^{\prime}\right) \operatorname{Diag}\left(G_{k+1}\right) \mathbf{1}^{\boldsymbol{\top}}\right]}{\left(F_{k}^{\theta, a_{1}} Q_{\theta} \operatorname{Diag}\left(G_{k+1}\right) \mathbf{1}^{\boldsymbol{\top}}\right)^{2}} \times F_{k}^{\theta, a_{1}} Q_{\theta} \operatorname{Diag}\left(G_{k+1}\right),
\end{aligned}
$$

and a similar equation holds for $\partial_{\beta} F_{k+1}^{\theta, a_{1}}$.

In Section 4.3, we provide an illustration of the numerical performances of the maximum likelihood estimator in this setup. Note that second order derivatives of the prediction filter and thus the log-likelihood are obtained similarly. These are used to estimate the asymptotic covariance matrix of the MLE in Section 4.3.

To conclude this section, note that the regularity Assumptions 3 and 5 are satisfied, as well as the identifiability Assumption 4 , as soon as $a_{1} \neq a_{2}$ and $\alpha \neq \beta$. As a consequence, Theorems 3.4 and 3.5 are valid in this setup.

Example 3 (DNA unzipping). We consider the context of DNA unzipping studied in [7] where the goal is the sequencing of a molecule (see also [6]). The physical experiment consists in observing many different unzippings of a DNA molecule which, due to its physical properties, may naturally (re)-zip. In this context, the random walk $\mathbf{X}$ represents the position of the fork at each time $t$ of the experiment, or equivalently the number of currently unzipped bases of the molecule. In the previous works, the authors are interested in the observation of many finite trajectories of the random walk in this random environment. Here, we consider the different problem of a single unzipping of a sufficiently long molecule.

Let $\mathcal{A}=\{A, C, G, T\}$ denote the finite nucleotide alphabet. The sequence of bases $\left\{b_{x}\right\}_{1 \leq x \leq n} \in \mathcal{A}^{n}$ of the (finite length) molecule are unknown and induce a specific environment that will be considered as random. More precisely, the conditional transitions of the random walk are given by

$$
\omega_{x}=\frac{1}{1+\exp (\beta g(x, x+1))} .
$$

where $g(x, x+1):=g_{0}\left(b_{x}, b_{x+1}\right)-g_{1}(f)$. The known parameter $g_{1}(f)$ is the work to stretch under a force $f$ the open part of the two strands, it can be adjusted but is constant during the unzipping. Parameter $\beta>0$ is also known and proportional to the inverse of temperature. The quantity $g_{0}\left(b_{x}, b_{x+1}\right)$ is the binding energy that takes into account additional stacking effects and therefore depends on the base values at the $(x+1)$ th and also at the $x$ th positions. Table 1 gives these binding energies at room temperature (see [7]). To take into account this dependence between energies, we assume that $\left\{g_{0}(x):=g_{0}\left(b_{x}, b_{x+1}\right)\right\}_{x \geq 1}$ is a Markov chain. With this assumption and since the mapping $g_{0}(x) \mapsto \omega_{x}$ is one-to-one, $\boldsymbol{\omega}=\left(\omega_{x}\right)_{x \geq 1}$ is Markov as well. The parameter of the model is thus the transition matrix $Q_{\theta}$ between the binding energies. Note that while the set of dinucleotides 
TABLE 1. Binding free energies (units of $k_{B} T$ ).

\begin{tabular}{ccccc}
\hline$g_{0}$ & $\mathrm{~A}$ & $\mathrm{~T}$ & $\mathrm{C}$ & $\mathrm{G}$ \\
\hline $\mathrm{A}$ & 1.78 & 1.55 & 2.52 & 2.22 \\
$\mathrm{~T}$ & 1.06 & 1.78 & 2.28 & 2.54 \\
$\mathrm{C}$ & 2.54 & 2.22 & 3.14 & 3.85 \\
$\mathrm{G}$ & 2.28 & 2.52 & 3.90 & 3.14 \\
\hline
\end{tabular}

has cardinality 16, function $g_{0}$ takes only 10 different values. So random environment $\boldsymbol{\omega}$ takes values in $S$ with cardinality 10 and the underlying transition matrix $Q_{\theta}$ (for the binding energies) is of size $10 \times 10$ but has many zero entries.

The ballistic condition is not difficult to satisfy. Indeed, we have

$$
\tilde{\omega}_{x}=\exp \left(\beta\left(g_{0}(x)-g_{1}(f)\right)\right)
$$

and $g_{1}$ is increasing with $f$. Thus we may choose $f$ such that $g_{1}$ is large enough to ensure either $\mathbf{E}^{\theta}\left(\tilde{\omega}_{0}\right)<1$ if the sequence $\left\{g_{0}(x)\right\}_{x \geq 1}$ is only i.i.d. or to ensure $\mathbf{E}^{\theta}\left(\log \tilde{\omega}_{0}\right)<0$ when the sequence $\left\{g_{0}(x)\right\}_{x \geq 1}$ is not independent. In both cases, this ensures the ballistic regime.

In this context and for a long enough sequence, we can estimate the matrix $Q_{\theta}$ of the transitions between the different binding energies, as well as $\mu_{\theta}$ which gives the frequencies of appearance of the binding energies. In turn, this also gives the frequencies of appearance of certain base pairs thanks to Table 1. Since both parameter space $\Theta$ and state space $S$ are finite, Assumptions 2, 3 and 5 are satisfied. This is also the case for identifiability Assumption 4. As a consequence, Theorems 3.4 and 3.5 are valid in this setup.

Example 4 (Auto-regressive environment). Let $y_{0} \sim \mu_{\theta}$ and for any $n \geq 0$, we let $y_{n+1}=\alpha y_{n}+u_{n}$ where $\alpha \in \mathbb{R}$ and $\left(u_{n}\right)_{n \geq 0}$ is an i.i.d. sequence. Fix some $\varepsilon>0$. The environment $\boldsymbol{\omega}$ is defined on $S=[\varepsilon, 1-\varepsilon]$ through a truncated logistic function

$$
\omega_{n}=\phi_{\varepsilon}\left(y_{n}\right):= \begin{cases}\mathrm{e}^{y_{n}}\left(1+\mathrm{e}^{y_{n}}\right)^{-1} & \text { if } \mathrm{e}^{y_{n}}\left(1+\mathrm{e}^{y_{n}}\right)^{-1} \in S, \\ \varepsilon & \text { if } \mathrm{e}^{y_{n}}\left(1+\mathrm{e}^{y_{n}}\right)^{-1} \leq \varepsilon, \\ 1-\varepsilon & \text { if } \mathrm{e}^{y_{n}}\left(1+\mathrm{e}^{y_{n}}\right)^{-1} \geq 1-\varepsilon .\end{cases}
$$

A time reversibility condition on first-order autoregressive processes is studied in [34]. If we assume that $u_{n}$ has Gaussian distribution, say $u_{n} \sim \mathcal{N}\left(\mu, \sigma^{2}\right)$, then for any value $|\alpha|<1$, it is easily seen that there exists a stationary density $\mu_{\theta}$ for $\left(y_{n}\right)_{n \geq 0}$ given by

$$
\forall y \in \mathbb{R}, \quad \mu_{\theta}(y)=\left(\frac{1-\alpha^{2}}{2 \pi \sigma^{2}}\right)^{1 / 2} \exp \left[-\frac{1-\alpha^{2}}{2 \sigma^{2}}\left\{y-\frac{\mu}{1-\alpha}\right\}^{2}\right]
$$

where $\theta=\left(\alpha, \mu, \sigma^{2}\right)$ is the model parameter. Moreover, the process $\left(y_{n}\right)_{n \geq 0}$ is reversible w.r.t. this stationary distribution. Then $\left(\omega_{n}\right)_{n \geq 0}$ is also stationary and time reversible.

Note that the inverse function $\phi_{\varepsilon}^{-1}: S \rightarrow[\log (\varepsilon /(1-\varepsilon)), \log ((1-\varepsilon) / \varepsilon)]$ is well defined and given by

$$
\forall a \in S, \quad \phi_{\varepsilon}^{-1}(a)=\log \left(\frac{a}{1-a}\right) .
$$

The transience condition writes $\mathbf{E}^{\theta}\left(y_{0}\right)>0$ or equivalently $\mu>0$. As soon as $\alpha \neq 0$, the sequences $\left(y_{n}\right)_{n}>0$ and thus also $\left(\omega_{n}\right)_{n \geq 0}$ are non independent and the existence of a unique stationary distribution implies the ballistic regime from transience assumption (Lem. 6.1 in [2]). Now, the transition density of $\boldsymbol{\omega}$ is given by

$$
q_{\theta}(a, b)=\frac{1}{\sqrt{2 \pi} \sigma b(1-b)} \exp \left(-\frac{1}{2 \sigma^{2}}\left(\phi_{\varepsilon}^{-1}(b)-\alpha \phi_{\varepsilon}^{-1}(a)-\mu\right)^{2}\right) .
$$




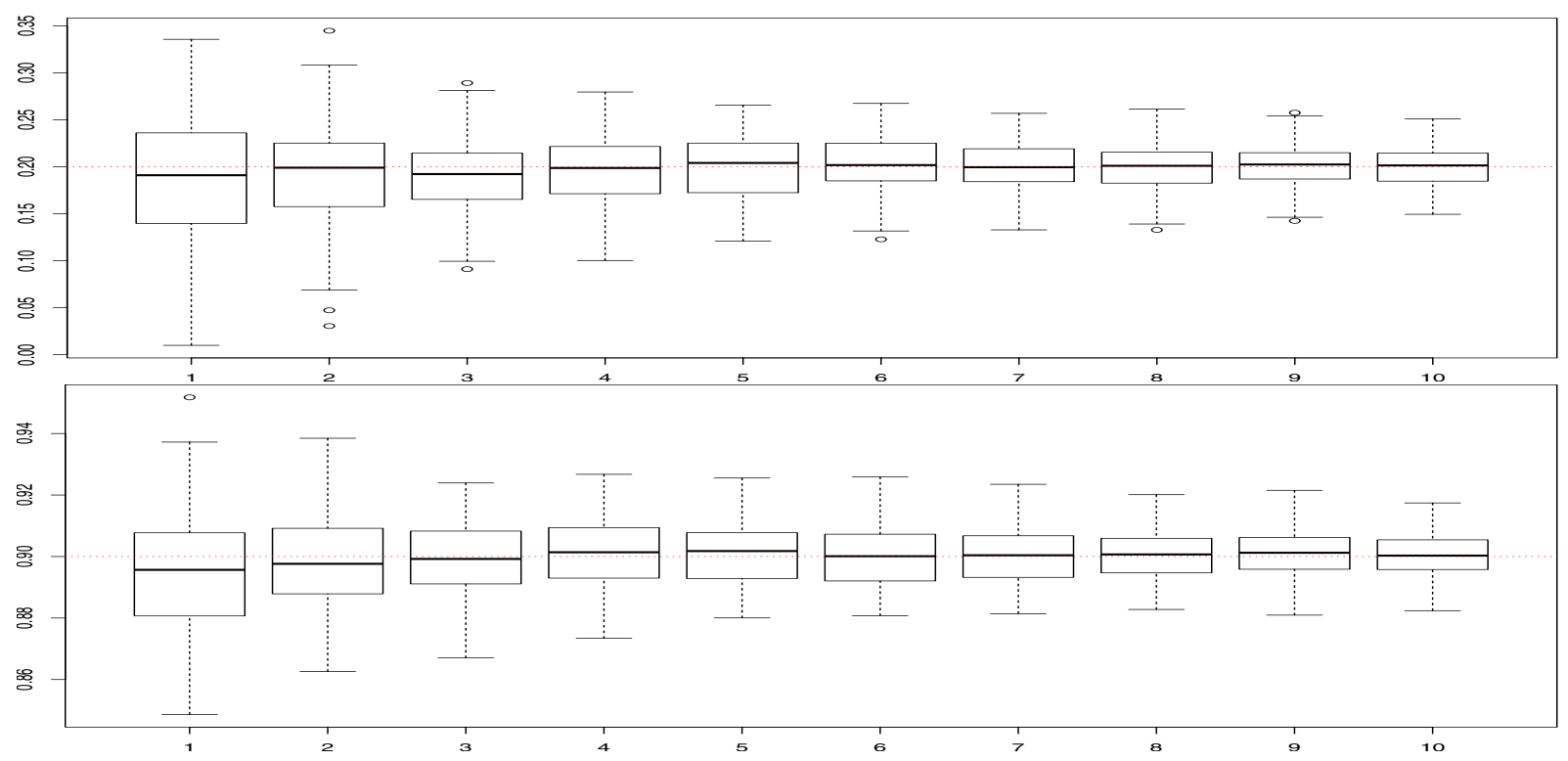

Figure 1. Boxplots of MLE obtained from $M=100$ iterations and for values $n$ ranging in $\left\{10^{3} k ; 1 \leq k \leq 10\right\}$ ( $x$-axis indicates the value $k$ ). First and second panel display estimation of $\alpha^{\star}$ and $\beta^{\star}$, respectively. The true values are indicated by horizontal lines.

As a consequence, Assumption 2 is satisfied as soon as $\sigma^{2}$ and $\mu$ are bounded. Thus we assume that the parameter space satisfies

$$
\Theta=\mathcal{A} \times\left[\mu_{\min }, \mu_{\max }\right] \times\left[\sigma_{\min }, \sigma_{\max }\right]
$$

where $\mathcal{A}$ is a compact subset of $(-1,1)$ and the constants satisfy $\mu_{\min }>C(\varepsilon)+\sigma_{\max }^{2} / 2$ and $\sigma_{\min }>0$. Moreover, regularity Assumptions 3 and 5 are also satisfied, as well as identifiability Assumption 4. As a consequence, Theorems 3.4 and 3.5 are valid in this setup.

\subsection{Numerical performance}

In this section, we illustrate our results in the simple case of Example 2. We start by describing the experiment. The support of the environment is fixed to $S=\{0.4,0.8\}$. The true parameter value is chosen as $\left(\alpha^{\star}, \beta^{\star}\right)=$ $(0.2,0.9)$. These choices ensure transience of the walk as well as ballistic regime. Next, we repeat 100 times the following procedure. We simulate a RWRE under the model described in Example 2 with parameter values $\left(\alpha^{\star}, \beta^{\star}\right)$ and stop it successively at the hitting times $T_{n}$, with $n \in\left\{10^{3} k ; 1 \leq k \leq 10\right\}$. For each value of $n$, the likelihood is computed as detailed in the previous section and we compute the MLE $\left(\hat{\alpha}_{n}, \hat{\beta}_{n}\right)$ through numerical optimisation of this likelihood. The likelihood optimisation procedure is performed according to the "L-BFGSB" method of [13]. It is worth mentioning that the length of the random walk is not $n$ but rather $T_{n}$, a quantity that is much larger in practice, see e.g. Section 5.2 in [18]. Figure 1 shows the boxplots of the MLE obtained from $M=100$ iterations of the procedure and increasing values of $n$. The red horizontal dotted line shows the true parameter value. As expected, the MLE converges to the true value as $n$ increases.

We further explore estimation of the asymptotic covariance matrix through the Hessian of the log-likelihood according to (3.6). Note that the true value $I\left(\theta^{\star}\right)^{-1}$ is unknown as there is no constructive form of the Fisher information for this model. However, this true value may be approximated by the empirical covariance matrix obtained from running the above experiment with $M=100$ iterations. Figure 2 shows the boxplots of the entries 

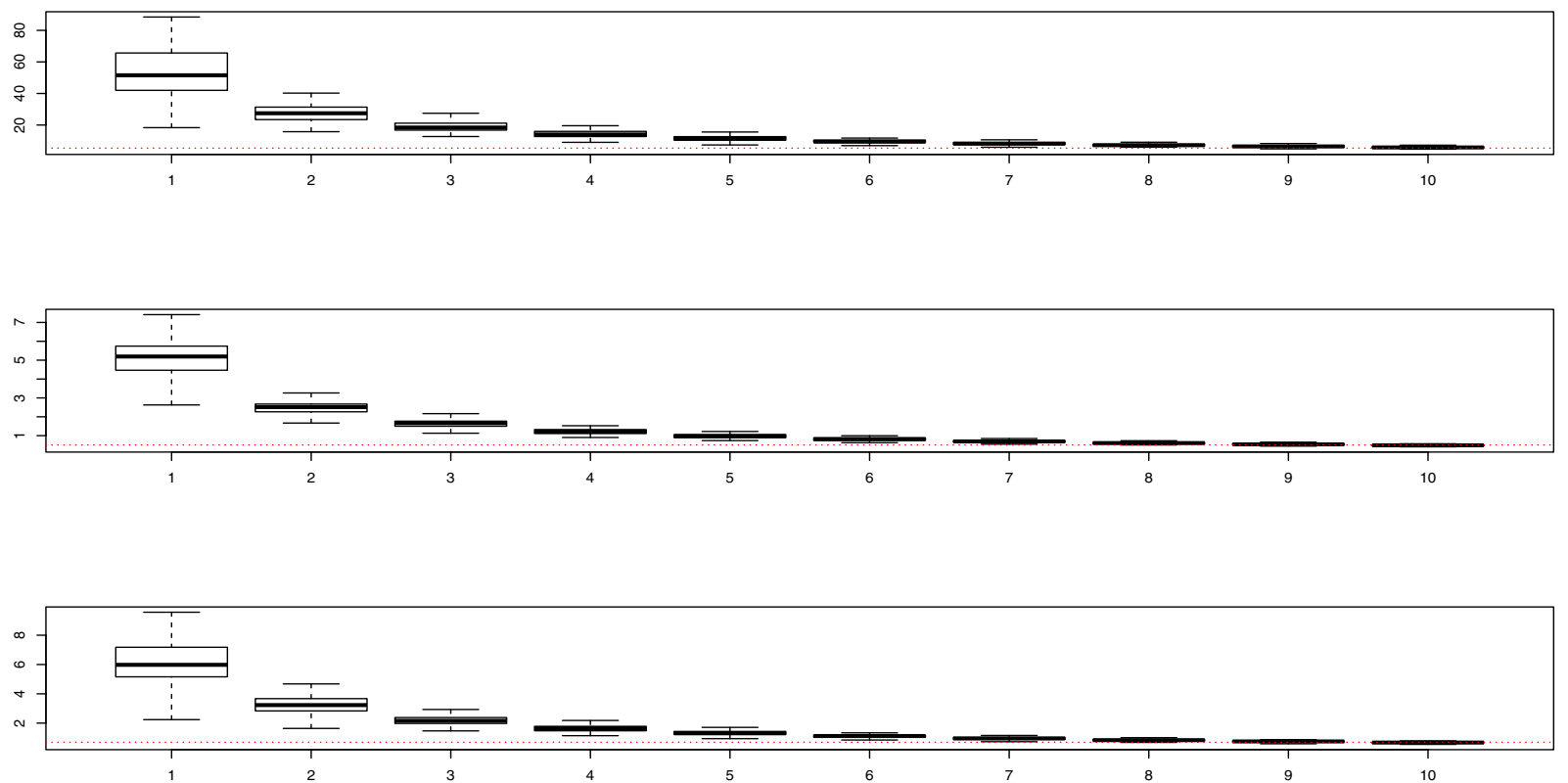

Figure 2. Boxplots of the entries of $\hat{\Sigma}_{n}$ obtained from $M=100$ iterations and for values $n$ ranging in $\left\{10^{3} k ; 1 \leq k \leq 10\right\}$ ( $x$-axis indicates the value $k$ ). From top to bottom: second derivative with respect to $\alpha$, second derivative with respect to $\beta$ and second derivative with respect to $\alpha$ and $\beta$. The red dotted line is the empirical estimate of the covariance matrix entries obtained from $M=100$ iterations for the largest value $n=10000$. From top to bottom: $\widehat{\operatorname{Var}}\left(\hat{\alpha}_{n}\right), \widehat{\operatorname{Var}}\left(\hat{\beta}_{n}\right), \widehat{\operatorname{Cov}}\left(\hat{\alpha}_{n}, \hat{\beta}_{n}\right)$. (Color online).

of the opposite normalized Hessian of the log-likelihood at the estimated parameter value, namely

$$
\hat{\Sigma}_{n}:=-\frac{1}{n} \partial_{\theta}^{2} \ell_{n}(\hat{\theta})
$$

obtained by iterating the procedure $M=100$ times. The red horizontal dotted line does not represent the entries of $I\left(\theta^{\star}\right)^{-1}$ (which remain unknown even if $\theta^{\star}$ is known) but rather the entries of the empirical covariance estimate matrix

$$
\widehat{\operatorname{Cov}}\left(\hat{\theta}_{n}\right):=\frac{1}{M} \sum_{i=1}^{M}\left(\hat{\theta}_{n}^{(i)}-\frac{1}{M} \sum_{i=1}^{M} \hat{\theta}_{n}^{(i)}\right)^{\top}\left(\hat{\theta}_{n}^{(i)}-\frac{1}{M} \sum_{i=1}^{M} \hat{\theta}_{n}^{(i)}\right),
$$

where $\hat{\theta}_{n}^{(i)}$ is the estimator obtained at $i$ th iteration. We choose the most accurate estimator obtained with $n=10000$. The results obtained are quite good.

To conclude this section, we consider the construction of confidence regions for $\left(\alpha^{\star}, \beta^{\star}\right)$. The asymptotic normality of the estimator $\hat{\theta}_{n}$ together with the estimation of the asymptotic variance $I\left(\theta^{\star}\right)^{-1}$ leads to the following confidence region

$$
\mathcal{R}_{\gamma, n}:=\left\{\theta \in \Theta, n\left(\hat{\theta}_{n}-\theta\right)^{\top} \hat{\Sigma}_{n}\left(\hat{\theta}_{n}-\theta\right) \leq \chi_{1-\gamma}\right\},
$$

where $1-\gamma$ is the asymptotic confidence level and $\chi_{z}$ is the $z$ th quantile of the chi-square distribution with 2 degrees of freedom. Table 2 presents the empirical coverages obtained from these confidence regions $\mathcal{R}_{\gamma, n}$ with $M=100$ iterations and for $\gamma \in\{0.01,0.05,0.1\}$ and $n$ ranging in $\left\{10^{3} k ; 1 \leq k \leq 10\right\}$. For the values $n \leq 6000$ we observe that the confidence regions are too wide. However, for the large values $n \geq 9000$ the empirical coverages are quite good. 
TABLE 2. Empirical coverage of $(1-\gamma)$ asymptotic level confidence regions, with $\gamma \in$ $\{0.01,0.05,0.1\}$.

\begin{tabular}{cccc}
\hline$n$ & 0.01 & 0.05 & 0.1 \\
\hline 1000 & 1.00 & 1.00 & 1.00 \\
2000 & 1.00 & 1.00 & 1.00 \\
3000 & 1.00 & 1.00 & 1.00 \\
4000 & 1.00 & 1.00 & 1.00 \\
5000 & 1.00 & 1.00 & 0.99 \\
6000 & 1.00 & 0.99 & 0.98 \\
7000 & 1.00 & 0.98 & 0.97 \\
8000 & 0.99 & 0.98 & 0.95 \\
9000 & 0.98 & 0.97 & 0.96 \\
10000 & 0.99 & 0.95 & 0.92 \\
\hline
\end{tabular}

\section{Proofs}

\subsection{Properties of the underlying HMM}

In this section, we investigate the properties of the bivariate process $\left\{\left(\breve{\omega}_{k}, Z_{k}\right)\right\}_{k \geq 0}$, namely we show that it is positive Harris recurrent and we exhibit its invariant distribution. Let us first define $\breve{R}$ for the time reversed environment $\breve{\boldsymbol{\omega}}$ similarly as $R$ from equation $(2.2)$ by

$$
\breve{R}=\left(1+\tilde{\omega}_{-1}+\tilde{\omega}_{-1} \tilde{\omega}_{-2}+\ldots\right) .
$$

We first remark that Condition (2.4) writes the same for $\boldsymbol{\omega}$ and $\breve{\boldsymbol{\omega}}$ so that environment $\breve{\boldsymbol{\omega}}$ is ballistic under Assumption 1 and thus $\mathbf{E}^{\theta}(\breve{R})<+\infty$. Moreover, under Assumptions 1 and 2, we obtain the following uniform ballistic condition on the time reversed environment

$$
1 \leq \inf _{a \in S} \mathbf{E}_{a}^{\theta}(\breve{R}) \leq \sup _{a \in S} \mathbf{E}_{a}^{\theta}(\breve{R}) \leq c_{+}<\infty
$$

for some positive and finite constant $c_{+}$. Indeed, the lower bound follows from $\breve{R} \geq 1$, by definition of $R$. Now, for any $a \in(0,1)$, we let $\tilde{a}=(1-a) / a$. The upper bound is obtained through

$$
\mathbf{E}_{a}^{\theta}(\breve{R})=1+\mathbf{E}_{a}^{\theta}\left[\tilde{\omega}_{-1} \mathbf{E}_{\omega_{-1}}^{\theta}(\breve{R})\right]=1+\int_{S} \tilde{b} \mathbf{E}_{b}^{\theta}(\breve{R}) \breve{q}_{\theta}(a, b) \mathrm{d} b \leq 1+\frac{(1-\varepsilon) \sigma_{+}}{\varepsilon \sigma_{-}} \mathbf{E}^{\theta}(\breve{R}),
$$

where the first equality above is the strong Markov property and the inequality uses both (3.2) and the lower bound (3.3) on the stationary distribution $\mu_{\theta}$.

The following proposition states the recurrence result on the Markov chain $\left\{\left(\breve{\omega}_{k}, Z_{k}\right)\right\}_{k \geq 0}$ and gives an expression for the density $\pi_{\theta}$ of the corresponding invariant distribution.

Proposition 5.1. Under Assumptions 1 and 2, the Markov chain $\left\{\left(\breve{\omega}_{k}, Z_{k}\right)\right\}_{k \geq 0}$ whose transition kernel is given by (2.9) is positive Harris recurrent and aperiodic with invariant density distribution $\pi_{\theta}$ given by

$$
\forall(a, x) \in S \times \mathbb{N}, \quad \pi_{\theta}(a, x)=\mu_{\theta}(a) \mathbf{E}_{a}^{\theta}\left(R^{-1}\left(1-R^{-1}\right)^{x}\right) .
$$

Proof. Note that $\pi_{\theta}$ is indeed a density. We first prove that it is the density of an invariant distribution. Thus we want to establish that for any $(b, y) \in S \times \mathbb{N}$, we have

$$
\pi_{\theta}(b, y)=\sum_{x \in \mathbb{N}} \int_{S} \pi_{\theta}(a, x) \Pi_{\theta}((a, x),(b, y)) \mathrm{d} a .
$$


We start by considering the right-hand side of the above equation where we input the expressions for density $\pi_{\theta}$ and kernel $\Pi_{\theta}$. We let

$$
\begin{aligned}
T & =\sum_{x \in \mathbb{N}} \int_{S} \pi_{\theta}(a, x) \Pi_{\theta}((a, x),(b, y)) \mathrm{d} a \\
& =\sum_{x \in \mathbb{N}} \int_{S} \mu_{\theta}(a) \mathbf{E}_{a}^{\theta}\left[R^{-1}\left(1-R^{-1}\right)^{x}\right]\left(\begin{array}{c}
x+y \\
x
\end{array}\right) \breve{q}_{\theta}(a, b) b^{x+1}(1-b)^{y} \mathrm{~d} a .
\end{aligned}
$$

From the definition of $\breve{q}_{\theta}$ and using Fubini's theorem for positive functions, we get

$$
\begin{aligned}
T & =\mu_{\theta}(b) \int_{S} q_{\theta}(b, a)(1-b)^{y} \sum_{x \in \mathbb{N}}\left(\begin{array}{c}
x+y \\
x
\end{array}\right) \mathbf{E}_{a}^{\theta}\left[R^{-1}\left(1-R^{-1}\right)^{x}\right] b^{x+1} \mathrm{~d} a \\
& =\mu_{\theta}(b) \int_{S} q_{\theta}(b, a)(1-b)^{y} \mathbf{E}_{a}^{\theta}\left[\frac{R^{-1} b}{\left[1-b\left(1-R^{-1}\right)\right]^{y+1}}\right] \mathrm{d} a \\
& =\mu_{\theta}(b) \int_{S} q_{\theta}(b, a) \mathbf{E}_{a}^{\theta}\left[\frac{1}{1+\tilde{b} R} \times\left(\frac{1-b}{1-b+b R^{-1}}\right)^{y}\right] \mathrm{d} a .
\end{aligned}
$$

Now, applying Markov's property and the definition of the shift operator, we obtain

$$
\begin{aligned}
T & =\mu_{\theta}(b) \mathbf{E}_{b}^{\theta}\left(\mathbf{E}_{\omega_{1}}^{\theta}\left[\frac{1}{1+\tilde{b} R} \times\left(\frac{1-b}{1-b+b R^{-1}}\right)^{y}\right]\right) \\
& =\mu_{\theta}(b) \mathbf{E}_{b}^{\theta}\left(\mathbf{E}_{b}^{\theta}\left[\frac{1}{1+\tilde{b} R} \times\left(\frac{\tilde{b} R}{1+\tilde{b} R}\right)^{y} \circ \tau^{1} \mid \mathcal{F}_{1}\right]\right) \\
& =\mu_{\theta}(b) \mathbf{E}_{b}^{\theta}\left(\mathbf{E}_{b}^{\theta}\left[\frac{1}{1+\tilde{b}+\tilde{b} \omega_{1}+\ldots} \times\left(\frac{\tilde{b}+\tilde{b} \tilde{\omega}_{1}+\ldots}{1+\tilde{b}+\tilde{b} \tilde{\omega}_{1}+\ldots}\right)^{y} \mid \mathcal{F}_{1}\right]\right) \\
& =\mu_{\theta}(b) \mathbf{E}_{b}^{\theta}\left(R^{-1}\left(1-R^{-1}\right)^{y}\right) .
\end{aligned}
$$

This concludes the validity of (5.3).

As the marginal process $\left\{\omega_{k}\right\}_{k \geq 0}$ is aperiodic, this is also the case for the time reversed marginal process $\left\{\breve{\omega}_{k}\right\}_{k \geq 0}$ and for the bivariate process $\left\{\left(\breve{\omega}_{k}, Z_{k}\right)\right\}_{k \geq 0}$. Following Theorem 9.1.8 in [33], we want to prove that the Markov chain $\left\{\left(\breve{\omega}_{k}, Z_{k}\right)\right\}_{k \geq 0}$ is $\psi$-irreducible for some probability measure $\psi$ and that there exists a petite set $C \in S \times \mathbb{N}$ and a function $V: S \times \mathbb{N} \rightarrow \mathbb{R}_{+}$, such that

(1) $\Delta V(a, x):=\Pi_{\theta} V(a, x)-V(a, x) \leq 0, \quad \forall(a, x) \notin C ;$

(2) $\forall N \in \mathbb{N}, V_{N}:=\{(a, x) \in S \times \mathbb{N} ; V(a, x) \leq N\}$ is a petite set.

For all $B \in \mathcal{B}(S \times \mathbb{N})$ and $i \in\{1,2\}$ let $\operatorname{pr}_{i}(B)$ be the projection of $B$ onto $S$ when $i=1$ and onto $\mathbb{N}$ when $i=2$. We also let $T_{B}$ be the first hitting time of the set $B$ by the chain $\left\{\left(\breve{\omega}_{k}, Z_{k}\right)\right\}_{k \geq 0}$. Thanks to Assumptions 2 and (3.2), we can write

$$
\begin{aligned}
\mathbb{P}_{(a, x)}^{\theta}\left(T_{B}<\infty\right) & \geq \int_{p r_{1}(B)} \sum_{y \in p r_{2}(B)}\left(\begin{array}{c}
x+y \\
x
\end{array}\right) b^{x+1}(1-b)^{y} \breve{q}_{\theta}(a, b) \mathrm{d} b \\
& \geq \frac{\sigma_{-}^{2}}{\sigma_{+}} \varepsilon^{x} \int_{p r_{1}(B)} \sum_{y \in p r_{2}(B)} b(1-b)^{y} \mathrm{~d} b .
\end{aligned}
$$

Hence the Markov's chain is $\varphi$-irreducible (see Sect. 4.2 in [33]), where the measure $\varphi$ defined on $\mathcal{B}(S \times \mathbb{N}$ ) by

$$
B \mapsto \varphi(B):=\int_{p r_{1}(B)} \sum_{y \in p r_{2}(B)} b(1-b)^{y} \mathrm{~d} b
$$


is a probability measure. From Proposition 4.2.2 in [33], the chain is also $\psi$-irreducible. Thanks to Assumption 2 again, we can easily see that for all $N \in \mathbb{N}$, the set $S \times \llbracket 1, N \rrbracket$ is a small set and as a consequence a petite set. Indeed, for any $(a, x) \in S \times \llbracket 1, N \rrbracket$ we have

$$
\Pi_{\theta}((a, x),(b, y)) \geq \frac{\sigma_{-}^{2}}{\sigma_{+}} b^{N+1}(1-b)^{y} \geq \frac{\sigma_{-}^{2}}{\sigma_{+}} \varepsilon^{N} b(1-b)^{y} .
$$

Let

$$
V(a, x)=x \mathbf{E}_{a}^{\theta}(\breve{R})=x \mathbf{E}_{a}^{\theta}\left(1+\tilde{\omega}_{-1}+\tilde{\omega}_{-1} \tilde{\omega}_{-2}+\ldots\right) .
$$

By using (5.2), function $V$ is finite. Moreover, we get that if $(a, x) \in V_{N}$, then $x \leq N$, which proves that for all $N \in \mathbb{N}$, the set $V_{N}$ is a petite set. Now, we consider

$$
\begin{aligned}
\Pi_{\theta} V(a, x) & =\int_{S} \sum_{y \in \mathbb{N}} y \mathbf{E}_{b}^{\theta}(\breve{R}) \Pi_{\theta}((a, x) ;(b, y)) \mathrm{d} b \\
& =\int_{S} \sum_{y \in \mathbb{N}} y \mathbf{E}_{b}^{\theta}(\breve{R})\left(\begin{array}{c}
x+y \\
x
\end{array}\right) b^{x+1}(1-b)^{y} \breve{q}_{\theta}(a, b) \mathrm{d} b \\
& =(x+1) \int_{S} \breve{q}_{\theta}(a, b)\left(\frac{1-b}{b}\right) \mathbf{E}_{b}^{\theta}(\breve{R}) \mathrm{d} b \\
& =(x+1) \mathbf{E}_{a}^{\theta}\left[\tilde{\omega}_{-1} \mathbf{E}_{\omega-1}^{\theta}(\breve{R})\right] \\
& =(x+1) \mathbf{E}_{a}^{\theta}\left[\tilde{\omega}_{-1} \mathbf{E}_{a}^{\theta}\left[\breve{R} \circ \tau^{1} \mid \breve{\mathcal{F}}_{1}\right]\right] \\
& =(x+1) \mathbf{E}_{a}^{\theta}\left[\tilde{\omega}_{-1}\left(1+\tilde{\omega}_{-2}+\tilde{\omega}_{-2} \tilde{\omega}_{-3}+\ldots\right)\right] \\
& =(x+1) \mathbf{E}_{a}^{\theta}(\breve{R}-1) .
\end{aligned}
$$

Note also that $\left(c_{+}-1\right) \geq \mathbf{E}_{a}^{\theta}(\breve{R}-1)>0$. As a consequence, for all $(a, x) \notin S \times \llbracket 0, c_{+}-1 \rrbracket$ we have $\Pi_{\theta} V(a, x) \leq$ $V(a, x)$. This concludes the proof of the proposition.

\subsection{Proof of consistency}

Consistency of the maximum likelihood estimator is given by Theorem 1 in [22] for the observations generated under stationary distribution and then extended by Theorem 5 in the same reference for a general initial distribution case. Both results are established under some assumptions that we now investigate in our context. Note that our process is not stationary since it starts from $\left(\omega_{0}, Z_{0}\right) \sim \mu_{\theta^{\star}} \otimes \delta_{0}$. Thus, we rely on Theorem 5 from [22] to establish the properties of our estimator. We show that our assumptions on the RWRE ensure the general assumptions on the autoregressive process with Markov regime needed to establish the consistency of the MLE (assumptions (A1) to (A5) in [22]).

First, Assumption 2 is sufficient to ensure assumption (A1) from [22]. Indeed, Assumption 2 implies inequalities (3.4) which correspond exactly to part (a) of (A1) on transition $\breve{q}_{\theta}$. Moreover, statement (b) of (A1) writes in our case as

$$
\forall(x, y) \in \mathbb{N}^{2}, \quad \int_{S} g_{a}(x, y) \mathrm{d} a=\left(\begin{array}{c}
x+y \\
x
\end{array}\right) \int_{S} a^{x+1}(1-a)^{y} \mathrm{~d} a
$$

positive and finite, which is automatically satisfied here.

Assumption (A2) from [22] requires that the transition kernel density $\Pi_{\theta}$ of the Markov chain $\left\{\left(\breve{\omega}_{k}, Z_{k}\right)\right\}_{k \geq 0}$ defined by (2.9) is positive Harris recurrent and aperiodic. In Proposition 5.1, we proved that this is satisfied as soon as this is the case for the environment kernel $q_{\theta}$ (namely Assumption 2) and under the ballistic Assumption 1 on the RWRE. Let us recall that $\overline{\mathbb{P}}^{\theta}$ and $\overline{\mathbb{E}}^{\theta}$ are the probability and expectation induced when considering the chain $\left\{\left(\breve{\omega}_{k}, Z_{k}\right)\right\}_{k \geq 0}$ under its stationary distribution $\pi_{\theta}$.

With the ballistic condition, we obtain assumption (A3) from [22], as stated in the following proposition. 
Proposition 5.2. Under Assumptions 1 and 2, we have

$$
\sup _{(x, y) \in \mathbb{N}^{2}} \sup _{a \in S} g_{a}(x, y)<+\infty \text { and } \overline{\mathbb{E}}^{\theta}\left[\log \int_{S} g_{a}\left(Z_{0}, Z_{1}\right) \mathrm{d} a\right]<+\infty .
$$

Proof. The first condition is satisfied according to the definition of $g$ given in (2.8). Moreover, we have

$$
\log \int_{S} g_{a}\left(Z_{0}, Z_{1}\right) \mathrm{d} a=\log \left(\begin{array}{c}
Z_{0}+Z_{1} \\
Z_{0}
\end{array}\right)+\log \int_{S} a^{Z_{0}+1}(1-a)^{Z_{1}} \mathrm{~d} a .
$$

Relying on Stirling's approximation, we have

$$
\log \left(\begin{array}{c}
Z_{0}+Z_{1} \\
Z_{0}
\end{array}\right)=Z_{0} \log \left(1+\frac{Z_{1}}{Z_{0}}\right)+Z_{1} \log \left(1+\frac{Z_{0}}{Z_{1}}\right)+O_{P}\left(\log \left(Z_{0}+Z_{1}\right)\right),
$$

where $O_{P}(1)$ stands for a sequence that is bounded in probability. Thus we can write

$$
\log \left(\begin{array}{c}
Z_{0}+Z_{1} \\
Z_{0}
\end{array}\right) \leq Z_{0}+Z_{1}+O_{P}\left(\log \left(Z_{0}+Z_{1}\right)\right) .
$$

Moreover, under assumption (3.2), we have

$$
|S| \times\left[\left(Z_{0}+1\right) \log \varepsilon+Z_{1} \log \varepsilon\right] \leq \log \int_{S} a^{Z_{0}+1}(1-a)^{Z_{1}} \mathrm{~d} a \leq\left(Z_{0}+1\right) \log (1-\varepsilon)+Z_{1} \log (1-\varepsilon),
$$

where $|S|$ denotes either the Lebesgue's measure of $S$ or its cardinality when $S$ is discrete. As a conclusion, as soon as $\overline{\mathbb{E}}^{\theta}\left(Z_{0}\right)<+\infty$, the second statement in the proposition is satisfied. Now, from the definition of $\pi_{\theta}$ given in Proposition 5.1, we get

$$
\overline{\mathbb{E}}^{\theta}\left(Z_{0}\right)=\sum_{x \in \mathbb{N}} \int_{S} x \mu_{\theta}(a) \mathbf{E}_{a}^{\theta}\left(R^{-1}\left(1-R^{-1}\right)^{x}\right) \mathrm{d} a=\int_{S} \mu_{\theta}(a) \mathbf{E}_{a}^{\theta}(R-1) \mathrm{d} a=\mathbf{E}^{\theta}(R)-1,
$$

which is finite thanks to Assumption 1.

Assumption 3 on $q_{\theta}$ is sufficient to ensure (A4) from [22] on $\breve{q}_{\theta}$.

Now, we let $\overline{\mathbb{P}}^{\theta, Z}$ denote the marginal of the distribution $\overline{\mathbb{P}}^{\theta}$ on the set $\mathbb{N}^{\mathbb{N}}$ (corresponding to the second marginal). In order to ensure identifiability of the model (assumption (A5) in [22]), we require identifiability of the parameter from the distribution of the environment (Assumption 4 in our work).

Lemma 5.3. Under Assumption 4, the autoregressive process with Markov's regime has identifiable parameter, i.e.

$$
\forall \theta, \theta^{\prime} \in \Theta, \quad \theta=\theta^{\prime} \Longleftrightarrow \overline{\mathbb{P}}^{\theta, Z}=\overline{\mathbb{P}}^{\theta^{\prime}, Z} .
$$

Proof. We prove that $\theta$ is uniquely defined from $\overline{\mathbb{P}}^{\theta, Z}$. The knowledge of the distribution $\overline{\mathbb{P}}^{\theta, Z}$ means that for any $n \in \mathbb{N}$, any sequence $z_{0}, \ldots, z_{n} \in \mathbb{N}^{n+1}$, we know the quantity

$$
\overline{\mathbb{P}}^{\theta}\left(\left(Z_{0}, \ldots, Z_{n}\right)=\left(z_{0}, \ldots, z_{n}\right)\right)=\int_{S} \ldots \int_{S} \pi_{\theta}\left(a_{0}, z_{0}\right) \prod_{i=1}^{n} \breve{q}_{\theta}\left(a_{i-1}, a_{i}\right) \prod_{i=1}^{n} g_{a_{i}}\left(z_{i-1}, z_{i}\right) \mathrm{d} a_{0} \ldots \mathrm{d} a_{n} .
$$

Since $g$ does not depend on $\theta$ and is positive, if we assume that $\overline{\mathbb{P}}^{\theta, Z}=\overline{\mathbb{P}}^{\theta^{\prime}, Z}$ we obtain from the above expression that

$$
\pi_{\theta}\left(a_{0}, z_{0}\right) \prod_{i=1}^{n} \breve{q}_{\theta}\left(a_{i-1}, a_{i}\right)=\pi_{\theta^{\prime}}\left(a_{0}, z_{0}\right) \prod_{i=1}^{n} \breve{q}_{\theta^{\prime}}\left(a_{i-1}, a_{i}\right),
$$

almost surely (w.r.t. the underlying measure on $S^{n+1}$ ). Noting that Assumption 4 can be formulated on $q_{\theta}$ or on $\breve{q}_{\theta}$ equivalently, this implies $\theta=\theta^{\prime}$. 
Now a direct application from Theorem 5 in [22] combined with our previous developments establishes that under Assumptions 2 to 4 , the maximum likelihood estimator $\hat{\theta}_{n}$ converges $\mathbb{P}^{\star}$-almost surely to the true parameter value $\theta^{\star}$ as $n$ tends to infinity.

\subsection{Proof of asymptotic normality}

Applying Theorem 6 from [22] and using that in our case, their assumptions (A6) to (A8) are satisfied for $\breve{q}_{\theta}$ under our Assumption 5, we obtain the weak convergence of the conditional score to a Gaussian distribution, as soon as the asymptotic variance is defined, which means as soon as the Fisher information matrix is invertible.

Acknowledgements. We would like to thank an anonymous referee who made interesting comments on our work.

\section{REFERENCES}

[1] O. Adelman and N. Enriquez, Random walks in random environment: what a single trajectory tells. Israel J. Math. 142 (2004) 205-220.

[2] S. Alili. Asymptotic behaviour for random walks in random environments. J. Appl. Probab. 36 (1999) $334-349$.

[3] P. Andreoletti. On the concentration of Sinai's walk. Stochastic Process. Appl. 116 (2006) 1377-1408.

[4] P. Andreoletti, Almost sure estimates for the concentration neighborhood of Sinai's walk. Stochastic Processes Appl. 117 (2007) 1473-1490

[5] P. Andreoletti, On the estimation of the potential of Sinai's RWRE. Braz. J. Probab. Stat. 25 (2011) 121-144.

[6] P. Andreoletti and R. Diel, DNA unzipping via stopped birth and death processes with unknown transition probabilities. Appl. Math. Res. eXpress 2012 (2012) 184-208.

[7] V. Baldazzi, S. Cocco, E. Marinari and R. Monasson, Inference of DNA sequences from mechanical unzipping: an ideal-case study. Phys. Rev. Lett. 96 (2006) 128-102.

[8] L.E. Baum and T. Petrie, Statistical inference for probabilistic functions of finite state Markov chains. Ann. Math. Statist. 37 (1966) 1554-1563.

[9] L.E. Baum, T. Petrie, G. Soules and N. Weiss, A maximization technique occurring in the statistical analysis of probabilistic functions of Markov chains. Ann. Math. Statist. 41 (1970) 164-171.

[10] P.J. Bickel and Y. Ritov, Inference in hidden Markov models. I: Local asymptotic normality in the stationary case. Bernoulli 2 (1996) 199-228.

[11] P.J. Bickel, Y. Ritov and T. Rydén, Asymptotic normality of the maximum-likelihood estimator for general hidden Markov models. Ann. Stat. 26 (1998) 1614-1635.

[12] L. Bogachev, Random walks in random environments. Edited by J.P. Francoise, G. Naber and S.T. Tsou. Encycl. Math. Phys. 4 (2006) 353-371.

[13] R.H. Byrd, P. Lu, J. Nocedal and C.Y. Zhu, A limited memory algorithm for bound constrained optimization. SIAM J. Sci. Comput. 16 (1995) 1190-1208.

[14] O. Cappé, E. Moulines and T. Rydén, Inference in hidden Markov models. Springer Ser. Statist. Springer, New York (2005).

[15] A. Chambaz and C. Matias, Number of hidden states and memory: a joint order estimation problem for Markov chains with Markov regime. ESAIM: PS 13 (2009) 38-50.

[16] A. Chernov, Replication of a multicomponent chain by the lightning mechanism. Biofizika 12 (1967) $297-301$.

[17] F. Comets, M. Falconnet, O. Loukianov and D. Loukianova, Maximum likelihood estimator consistency for recurrent random walk in a parametric random environment with finite support. Technical report. Preprint arXiv:1404.2551 (2014).

[18] F. Comets, M. Falconnet, O. Loukianov, D. Loukianova and C. Matias, Maximum likelihood estimator consistency for ballistic random walk in a parametric random environment. Stochastic Processes Appl. 124 (2014) 268-288.

[19] A.P. Dempster, N.M. Laird and D.B. Rubin, Maximum likelihood from incomplete data via the EM algorithm. J. R. Stat. Soc. Ser. B 39 (1977) 1-38.

[20] R. Douc and C. Matias, Asymptotics of the maximum likelihood estimator for general hidden Markov models. Bernoulli 7 (2001) 381-420.

[21] R. Douc, E. Moulines, J. Olsson and R. van Handel, Consistency of the maximum likelihood estimator for general hidden Markov models. Ann. Stat. 39 (2011) 474-513.

[22] R. Douc, É. Moulines and T. Rydén, Asymptotic properties of the maximum likelihood estimator in autoregressive models with Markov regime. Ann. Stat. 32 (2004) 2254-2304.

[23] Y. Ephraim and N. Merhav, Hidden Markov processes. Special issue on Shannon theory: perspective, trends, and applications. IEEE Trans. Inform. Theory 48 (2002) 1518-1569.

[24] M. Falconnet, A. Gloter and D. Loukianova, Maximum likelihood estimation in the context of a sub-ballistic random walk in a parametric random environment. Math. Methods Stat. 23 (2014) 159-175.

[25] M. Falconnet, D. Loukianova and C. Matias, Asymptotic normality and efficiency of the maximum likelihood estimator for the parameter of a ballistic random walk in a random environment. Math. Methods Stat. 23 (2014) 1-19. 
[26] V. Genon-Catalot and C. Laredo, Leroux's method for general hidden Markov models. Stochastic Processes Appl. 116 (2006) 222-243.

[27] B.D. Hughes, Random walks and random environments, Random environments. Vol. 2 of Oxford Science Publications. The Clarendon Press Oxford University Press, New York (1996).

[28] J.L. Jensen and N.V. Petersen, Asymptotic normality of the maximum likelihood estimator in state space models. Ann. Statist. 27 (1999) 514-535.

[29] H. Kesten, M.V. Kozlov and F. Spitzer, A limit law for random walk in a random environment. Compos. Math. 30 (1975) $145-168$.

[30] F. Le Gland and L. Mevel, Exponential forgetting and geometric ergodicity in hidden Markov models. Math. Control Signals Syst. 13 (2000) 63-93.

[31] B.G. Leroux, Maximum-likelihood estimation for hidden Markov models. Stochastic Process. Appl. 40 (1992) $127-143$.

[32] T.A. Louis, Finding the observed information matrix when using the EM algorithm. J. R. Stat. Soc. Ser. B 44 (1982) $226-233$.

[33] S. Meyn and R.L. Tweedie, Markov chains and stochastic stability, 2nd edition. Cambridge University Press, Cambridge (2009).

[34] H. Ōsawa, Reversibility of first-order autoregressive processes. Stochastic Processes Appl. 28 (1988) 61-69.

[35] P. Révész, Random walk in random and non-random environments, 2nd edition. World Scientific (2005).

[36] Z. Shi, Sinai's walk via stochastic calculus. Panoramas et Synthèses 12 (2001) 53-74.

[37] Y. Sinai, The limiting behavior of a one-dimensional random walk in a random medium. Theory Probab. Appl. 27 (1982) $247-258$.

[38] F. Solomon, Random walks in a random environment. Ann. Probab. 3 (1975) 1-31.

[39] D.E. Temkin, One-dimensional random walks in a two-component chain. Soviet Math. Dokl. 13 (1972) $1172-1176$.

[40] O. Zeitouni, Random walks in random environment. In Lectures on probability theory and statistics. Vol. 1837 of Lect. Notes Math. Springer, Berlin (2004) 189-312. 